\title{
IMPLICIT FUNCTIONS AND DIFFERENTIAL EQUATIONS IN GENERAL ANALYSIS*
}

BY

\author{
LAWRENCE M. GRAVES†
}

The chief purpose of this paper is to discuss some special cases of the implicit function theorems obtained by Hildebrandt and Graves in the paper entitled Implicit functions and their differentials in general analysis. $\ddagger$ In particular I wish to discuss a generalization of the notion of differential equation, combining ideas due to Hahn and to Carathéodory. $\$$ The equations are of the form

$$
f\left(r, \int_{r_{0}}^{r} g\left(r, r^{\prime}, y\left(r^{\prime}\right), x\right) d r^{\prime}, y(r), x\right)=0 .
$$

Here $x$ may represent both initial values and parameters, and the functions $f, g$, and $y$ are supposed to be bounded and measurable in $r$ and $r^{\prime}$. Imbedding and existence theorems for equations of this form are obtained in Parts VI and VII. As indicated by the paper of Hahn just referred to, such theorems find important applications in the calculus of variations. Special theorems relating to linear equations are found in Part VIII.

Many other special cases of our general theory have appeared in the literature from other writers. $\|$ In some of these cases it naturally occurs that the hypotheses of the writers must be strengthened in order to make their theories fit under our general theory. On the other hand, we should expect that only a limited part of any special theory will flow directly from

* Presented to the Society, May 1, 1926; received by the editors June 9, 1926.

$\dagger$ National Research Fellow in Mathematics.

‡ These Transactions, vol. 29 (1927), pp. 127-153. In the sequel this paper will be referred to as Paper I. Frequent reference will also be made to Paper II, Graves, Riemann integration and Taylor's theorem in general analysis, these Transactions, vol. 29 (1927), pp. 163-177; and Paper III, Graves, Some theorems concerning measurablefunctions, Bulletin of the American Mathematical Society, vol. 32 (1926), p. 529.

\& See Hahn, Monatshefte für Mathematik und Physik, vol. 14 (1903), pp. 326-332.

Carathéodory, Vorlesungen über reelle Funktionen, p. 666.

|| Cf. Hart, these Transactions, vol. 18 (1917), p. 125; vol. 23 (1922), p. 30; Annals of Mathematics, (2), vol. 24 (1922), p. 23.

Barnett, American Journal of Mathematics, vol. 44 (1922), p. 172.

Bliss, these Transactions, vol. 21 (1920), p. 79.

Cotton, Bulletin de la Société Mathématique de France, vol. 38 (1910), p. 144. 
our general theory. Such is obviously the case, for example, with regard to the theory of the Fredholm or the Volterra equation of the second kind. However, we have succeeded in obtaining a much more complete theory of the differentiability of the solutions than is exhibited in the previous treatments of many of the special cases.

It is found to be economical of time and effort, even in specializing our general theory, to state theorems that are still very general. Thus a large part of the paper is still concerned with general ranges and abstract spaces.

Part I lists some examples (mostly abstract) of linear metric spaces. Each space consists of a class of functions on a range $\mathfrak{B}$ to a linear metric space. Part II contains some miscellaneous theorems on differentials and compact sets. Part III discusses the nature of the solution of the equation $G(x, y)=y *$ on subspaces $\mathfrak{X}^{\prime}$ and $\mathfrak{Y}^{\prime}$ of the main spaces $\mathfrak{X}$ and $\mathfrak{Y}$. The theorems of Part III are applied in Part VI to discuss the continuity in $r$ of the solutions of equation (1). Part IV discusses a special mode of dependence of the function $G$ on $x$ and $y$, and obtains as a special case an extended implicit function theorem generalizing those given by Bolza, Bliss and Mason, Hadamard, and Hobson.* Part V discusses a method of extending the domain of definition of a function so as to preserve a Lipschitz condition. Parts VI and VII discuss imbedding and existence theorems for "differential" equations in general analysis analogous to equation (1). Equation (1) is taken up as a special case. Part VIII discusses special properties of the solutions of linear equations of the form

$$
K_{1}(y(r), r)=\int_{r_{0}}^{r} K_{2}(y(r), r) d r+c .
$$

My acknowledgments are due to Professor Hildebrandt for some of the theorems, which are noted in the text.

\section{EXAMPLES OF LINEAR METRIC SPACES}

It is worth noting that all the spaces considered here, as well as the examples given by other writers (cf. Fréchet, Banach, Hahn) have as elements, functions on a range $\mathfrak{P}$ to a given linear metric space. Professor M. H. Ingraham informs me that every linear metric space which can be wellordered, is isomorphic with a class of numerically valued functions.

* See Bolza, Vorlesungen über Variationsrechnung, p. 160-168.

Bliss and Mason, Fields of extremals in space, these Transactions, vol. 11 (1910), p. 326.

Hadamard, Leçons sur le Calcul des Variations, vol. I, pp. 497-502.

Hobson, Proceedings of the London Mathematical Society, vol. 14 (1915), p. 147. 
It is also worth remarking that the property of being complete remains isolated from the other properties. That is to say, in spaces of type $A$ or $A_{0}$, completeness is added by a separate postulate, and in spaces of type $B$, $C_{0}$, or $C_{n}$, completeness is present if it is so in the range of functional values. All the types of spaces mentioned are special cases of type $A$.

We follow the practice of Paper I in denoting by $\mathfrak{B}$ and $\mathfrak{P}^{\prime}$ general ranges or classes (not empty), and by $\sigma$ and $\sigma^{\prime}$, numerically valued functions on $\mathfrak{P}$ and $\mathfrak{B}^{\prime}$ respectively, which will enter as scale functions.

1. Postulationally limited classes. We have the following types.

Type A. A system ( $\mathfrak{B}, \mathfrak{B}, \mathfrak{A}, \mathfrak{V},\|\|)$, satisfying conditions 1.1-1.9:

1.1. $\mathfrak{B}$ is a linear metric space.

1.2. $\mathfrak{A}$ is the number system for $\mathfrak{B}$.

1.3. $\mathfrak{Y}$ is a class of functions on $\mathfrak{B}$ to $\mathfrak{B}$, containing at least two functions.

1.4. The sum function, $y_{1}+y_{2}$, is contained in $\mathfrak{D}$, for every $y_{1}$ and $y_{2}$.

1.5. The product function, $y a$, is contained in $\mathfrak{Y}$, for every element $y$ of $\mathfrak{Z}$ and every number $a$.

1.6. \|\| is a function on $\mathfrak{V}$ to the real non-negative part of $\mathfrak{A}$.

1.7. $\|y\|=0$ implies $y(p)=v *$ for every $p$, that is, $y=y *$.

1.8. $\left\|y_{1}+y_{2}\right\| \leqq\left\|y_{1}\right\|+\left\|y_{2}\right\|$ for every $y_{1}$ and $y_{2}$.

1.9. $\|y a\|=\|y\||a|$ for every $y$ and $a$.

The sum of two functions and the product of a function by a number are defined in the customary way. Then postulates 1.4 and 1.5 express the linear closure of the class $\mathfrak{Y}$. We use the same sign \|\| for the norm or modulus in the spaces $\mathfrak{B}$ and $\mathfrak{Y}$. This can bring no confusion, since $y(p)$ denotes an element of the space $\mathfrak{B}$, viz., the functional value of the function $y$ at $p$, so that by $\|y(p)\|$ we understand the norm in the space $\mathfrak{B}$, and by $\|y\|$ the norm in the space $\mathfrak{Y}$.

Type $A_{0}$. Let $\mathfrak{V}$ be a class of functions $y$ on $\mathfrak{B}$ to $\mathfrak{B}$, satisfying postulates 1.1 to 1.5 for type $A$, and let $\sigma$ be a scale function on $\mathfrak{B}$ to $\mathfrak{A}$. Suppose each function $y$ of $\mathfrak{V}$ is bounded $(\mathfrak{B} ; \sigma)$, i.e., for each $y$ there exists a constant $M_{y}$ such that $\|y(p)\| \leqq M_{y}|\sigma(p)|$ for every $p$. Then if we set $\|y\|=$ minimum effective $M_{y}, \mathfrak{Y}$ is a space of type $A$.

2. Classes of all functions satisfying certain conditions. We have the following types.

Type $B$. Corresponding to a given $\mathfrak{B}, \mathfrak{B}$, and $\sigma$, let $\mathfrak{Z}$ be the class of all functions $y$ on $\mathfrak{B}$ to $\mathfrak{B}$ which are bounded $(\mathfrak{B} ; \sigma)$. Then $\mathfrak{V}$ is a space of type $A_{0}$, if $\|y\|$ is defined as above. If $\mathfrak{B}$ is complete, so is $\mathfrak{Y}$. 
Spaces of types $C_{0}$ and $C_{n}$ are subspaces of spaces of type $B$. Note that to secure completeness in a space of type $C_{n}$, the definition of the norm function \|\| must be altered.

Type $C_{0}$. Let $\mathfrak{X}_{0}$ be a region of a metric space $\mathfrak{X}$, and let $\mathfrak{B}=\mathfrak{X}_{0} \mathfrak{B}^{\prime}$. Let the scale function $\sigma$ be independent of $x$, and let $\mathfrak{Z}$ be the corresponding space of type $B$. Let $\mathfrak{Z}^{(0)}$ be the subspace of $\mathfrak{Y}$ composed of all those functions $y$ which are continuous on $\mathfrak{X}_{0}$ uniformly $\left(\left[\mathfrak{X}_{0}\right] \mathfrak{B}^{\prime} ; \sigma\right)$. (The square brackets around $\mathfrak{X}_{0}$ indicate that it may be omitted from the range of uniformity.) Then $\mathfrak{V}^{(0)}$ is a linear metric space of type $A_{0}$, and if $\mathfrak{B}$ is complete, so is $\mathfrak{y}^{(0)}$.

Type $C_{n}$. Let the space $\mathfrak{X}$ be linear metric, and let $\mathfrak{Y}^{(n)}$ be the subspace of $\mathfrak{Y}$ composed of all those functions $y$ which are of class $\mathbb{E}^{(n)}$ on $\mathfrak{X}_{0}$ uniformly $\left(\left[\mathfrak{X}_{0}\right] \mathfrak{B}^{\prime} ; \sigma\right)$. Then $\mathfrak{V}^{(n)}$ is a linear metric space of type $A_{0}$, if the function \|\| is unaltered.

The conditions for completeness, however, are now more complicated. Consider first the case when $\mathfrak{X}_{0}$ is omitted from the range of uniformity for the differentiability properties. We assume that the space $\mathfrak{B}$ is complete, and restrict the space $\mathfrak{Y}^{(n)}$ further by supposing that for each function $y$, all the differentials up to and including the $n$th differential are modular uniformly $\left(\mathfrak{X}_{0} \mathfrak{B}^{\prime} ; \sigma\right)$. Furthermore we alter the norm or distance function as follows: let $N(y)$ denote the norm $\|y\|$ of the function $y$ regarded as a point of the space $\mathfrak{V}$ of type $B$, and let $M_{1}(y), M_{2}(y), \cdots, M_{n}(y)$ denote the moduli of the first $n$ differentials of $y$, respectively. Then $\|y\|$ is now defined to be the greatest of the numbers $N(y), M_{1}(y), \cdots, M_{n}(y)$. It is readily verified that $\mathfrak{V}^{(n)}$ is still a linear metric space. To show that it is complete, we proceed as follows.

Consider first the case $n=1$. Let $\left\{y_{m}\right\}$ be a sequence of functions of the space $\mathfrak{Y}^{(1)}$ satisfying the condition

$$
\lim _{\substack{m_{1}=\infty \\ m_{2}=\infty}}\left\|y_{m_{1}}-y_{m_{2}}\right\|=0 .
$$

Then by classic arguments we know that there exists a function $y$ of the space $\mathfrak{V}^{(0)}$ (type $C_{0}$ ) such that

$$
\lim _{m=\infty} y_{m}=y \text { uniformly }\left(\mathfrak{X}_{0} \mathfrak{P}^{\prime} ; \sigma\right) .
$$

Similarly there exists a function $z\left(x, p^{\prime} ; d x\right)$ on $\mathfrak{X}_{0} \mathfrak{P}^{\prime} \mathfrak{X}$ to $\mathfrak{B}$ which is continuous on $\mathfrak{X}_{0}$ uniformly $\left(\mathfrak{P}^{\prime} \mathfrak{X} ; \sigma\|d x\|\right)$, modular on $\mathfrak{X}$ uniformly $\left(\mathfrak{X}_{0} \mathfrak{B}^{\prime} ; \sigma\right)$, and such that

$$
\lim _{m=\infty} d y_{m}=z \text { uniformly }\left(\mathfrak{X}_{0} \mathfrak{P}^{\prime} \mathfrak{X} ; \sigma\|d x\|\right) \text {. }
$$


Since $z$ is the limit of functions distributive in $d x, z$ is also distributive in its argument $d x$. It remains only to verify the third property in the definition of the class $\mathfrak{G}^{\prime}$. For convenience we now omit to write the argument p. The difference

$$
R\left(x, x_{0}\right)\left\|x-x_{0}\right\|=y(x)-y\left(x_{0}\right)-z\left(x_{0} ; x-x_{0}\right)
$$

is the sum of the following five terms:

$$
\begin{aligned}
& R_{1}=y(x)-y_{m}(x), \\
& R_{2}=y_{m}\left(x_{0}\right)-y\left(x_{0}\right), \\
& R_{3}=y_{m}(x)-y_{m}\left(x_{0}\right)-y_{q}(x)+y_{q}\left(x_{0}\right), \\
& R_{4}=y_{q}(x)-y_{q}\left(x_{0}\right)-d y_{q}\left(x_{0} ; x-x_{0}\right), \\
& R_{5}=d y_{q}\left(x_{0} ; x-x_{0}\right)-z\left(x_{0} ; x-x_{0}\right) .
\end{aligned}
$$

By Taylor's theorem, ${ }^{*}$ we can reduce $R_{3}$ to the form

$$
R_{3}=\int_{0}^{1}\left[d y_{m}\left(x_{0}+\left(x-x_{0}\right) r, x-x_{0}\right)-d y_{q}\left(x_{0}+\left(x-x_{0}\right) r, x-x_{0}\right)\right] d r,
$$

valid for all points $x$ in a neighborhood $\left(x_{0}\right)_{a}$ contained in $\mathfrak{X}_{0}$. From this we obtain the inequality

$$
\left\|R_{3}\right\| \leqq \int_{0}^{1}\left\|d y_{m}-d y_{q}\right\| d r \leqq \epsilon|\sigma|\left\|x-x_{0}\right\|,
$$

holding for all values of $m \geqq q$, where $q$ is an integer sufficiently large, and for all points $x$ in $\left(x_{0}\right)_{a}$. We use also the fact that the convergence of the sequence $\left\{d y_{m}\right\}$ is uniform $\left(\mathfrak{X}_{0} \mathfrak{P}^{\prime} \mathfrak{X} ; \sigma\|d x\|\right)$. For the same $q$ we have

$$
\left\|R_{5}\right\| \leqq \epsilon|\sigma|\left\|x-x_{0}\right\| \text {. }
$$

Since $y_{q}$ is of class $\mathfrak{C}^{\prime}$, we have

$$
\left\|R_{4}\right\| \leqq \epsilon|\sigma|\left\|x-x_{0}\right\|
$$

for all points $x$ in a neighborhood $\left(x_{0}\right)_{b}$. The integer $m$ is still at our disposal. For each $x$ in $\left(x_{0}\right)_{b}$ we can choose $m$ so large that

$$
\left\|R_{1}+R_{2}\right\| \leqq \epsilon|\sigma|\left\|x-x_{0}\right\| .
$$

Hence, whenever $x$ is sufficiently near $x_{0}$, we have

$$
\left\|R\left(x, x_{0}\right)\right\| \leqq 4 \epsilon|\sigma|,
$$

* See Paper II. 
so that the function $y$ is actually of class $\mathfrak{S}^{\prime}$ on $\mathfrak{X}_{0}$ uniformly $\left(\mathfrak{B}^{\prime} ; \sigma\right)$, and has the function $z$ for its differential.

To prove our statements for all values of $n$, we use induction. We suppose that $\mathfrak{Y}^{(k)}$ is complete, and that we are concerned with a $\mathfrak{Y}^{(k+1)}$. Then a regular sequence in $\mathfrak{Y}^{(k+1)}$ is regular in $\mathfrak{Y}^{(k)}$, and hence has a limit in $\mathfrak{Y}^{(k)}$. The sequence of $(k+1)$ st differentials likewise has a limit, which is shown to be the first differential of the $k$ th differential $d^{k} y$ of $y$, with the requisite uniformity, in the same way as the function $z$ above was shown to be $d y$. The only difference is that the range $\mathfrak{B}^{\prime}$ is replaced by $\mathfrak{P}^{\prime} \mathfrak{X} \mathfrak{X} \ldots \mathfrak{X}$. Then $y$ is of class $\left(^{(k+1)}\right.$, by Lemma 14.1 of Paper I.

In the case when the functions $y$ of the space $\mathfrak{Y}^{(n)}$ are required to be of class $\mathfrak{S}^{(n)}$ uniformly $\left(\mathfrak{X}_{0} \mathfrak{P}^{\prime} ; \sigma\right)$, we must assume that the region $\mathfrak{X}_{0}$ is convex, in order to apply Taylor's theorem to the term $R_{3}$, and then the proof for completeness is practically the same as before.

Type $D_{0}$. Let $\mathfrak{X}^{(0)}$ be a measurable set of points in an $m$-dimensional euclidean space $\mathfrak{X}$. Then the class $\mathfrak{B}$ composed of all functions $v$ on $\mathfrak{X}^{(0)}$ to $\Re$ (i.e., single-real-valued on $\mathfrak{X}^{(0)}$ ) which are bounded and measurable on $\mathfrak{X}^{(0)}$, is a complete linear metric space, if we set $\|v\|=$ the upper bound of $|v(x)|$.

Type $D . \mathfrak{Z}$ is the space of type $B$ corresponding to a given range $\mathfrak{B}$ and a space $\mathfrak{B}$ of type $D_{0}$. This $\mathfrak{V}$ may also be regarded as a class of functions on $\mathfrak{X}^{(0)} \mathfrak{B}$ to $\Re$, and we have

$$
\|y\|=\text { upper bound of } \frac{|y(x, p)|}{|\sigma(p)|}
$$

for those values of $p$ for which $\sigma(p) \neq 0$ and for all $x$.

Type $D_{1}$. Let $\mathfrak{X}^{(1)}$ be a subset of the set $\mathfrak{X}^{(0)}$. Let $\mathfrak{Y}^{\prime}$ be the class of all functions $y$ of the space $\mathfrak{V}$ (of type $D$, regarded as on $\mathfrak{X}^{(0)} \mathfrak{B}$ to $\Re$ ), which are continuous at the points of the set $\mathfrak{X}^{(1)}$ uniformly $(\mathfrak{B} ; \sigma)$. The definition of $\|y\|$ is not changed. Then $\mathfrak{Y}^{\prime}$ is a complete linear metric space.

II. Miscellaneous theorems ON Differentials AND COMPACT SETS

3. In case $\mathfrak{Y}$ is a space of type $A$, i.e., a class of functions $y$ on $\mathfrak{B}$ to $\mathfrak{B}$, and $F$ is a function on a range $\mathfrak{P}^{\prime}$ to $\mathfrak{Y}$, then if $F\left(p^{\prime}\right)=y$, we denote $y(p)$ also by $F\left(p^{\prime} \mid p\right)$. Thus $F\left(p^{\prime} \mid p\right)$ denotes an element of the space $\mathfrak{B}$, and a function $G$ on $\mathfrak{B}^{\prime} \mathfrak{B}$ to $\mathfrak{B}$ is determined by setting

$$
F\left(p^{\prime} \mid p\right)=G\left(p^{\prime}, p\right)
$$

Conversely, if a function $G$ on $\mathfrak{B}^{\prime} \mathfrak{P}$ to $\mathfrak{B}$ has the right properties, the equation 
(3.1) determines a corresponding function $F$ on $\mathfrak{B}^{\prime}$ to $\mathfrak{Y}$. We shall have frequent use for the notation $F\left(p^{\prime} \mid p\right)$.

4. In this paragraph we consider a complete linear metric space $\mathfrak{V}$ of type $A_{0}$, and a region $\mathfrak{X}_{0}$ of a linear metric space $\mathfrak{X}$. Consider also a function $F$ on $\mathfrak{X}_{0} \mathfrak{P}^{\prime}$ to $\mathfrak{Y}$, and the corresponding function $G$ on $\mathfrak{X}_{0} \mathfrak{P}^{\prime} \mathfrak{B}$ to $\mathfrak{B}$ determined by $F\left(x, p^{\prime} \mid p\right)=G\left(x, p^{\prime}, p\right)$. Then we have

ThEOREM I. If the function $F$ is of class $\mathfrak{C}^{(n)}$ on $\mathfrak{X}_{0}$ uniformly $\left(\left[\mathfrak{X}_{0}\right] \mathfrak{B}^{\prime} ; \sigma^{\prime}\right)$, then the function $G$ is of class $\mathfrak{E}^{(n)}$ on $\mathfrak{X}_{0}$ uniformly $\left(\left[\mathfrak{X}_{0}\right] \mathfrak{B}^{\prime} \mathfrak{P} ; \sigma^{\prime} \sigma\right)$, and conversely.

The proof is made by direct application of the definition of the class $\mathbb{E}^{\prime}$, and induction.

5. Compact sets and uniformity. ${ }^{*}$ In case a real-valued function $F$ defined on a region $\mathfrak{X}_{0}$ of an $m$-dimensional euclidean space $\mathfrak{X}$ is of class $\mathfrak{E}^{\prime}$ on $\mathfrak{X}_{0}$, then $F$ is of class $\mathbb{E}^{\prime}$ on $\mathfrak{X}_{0}$ uniformly on every bounded and closed set $\mathfrak{X}^{(1)}$ contained in $\mathfrak{X}_{0}$. However, in this as in other uniformity theorems, the closure of the set $\mathfrak{X}^{(1)}$ is not the essential hypothesis.

In a metric space $\mathfrak{X}$, an infinite sequence of sets $\mathfrak{X}^{(m)}$ is said to have an accumulation point $x_{0}$ in case every neighborhood of $x_{0}$ contains points from an infinity of sets $\mathfrak{X}^{(m)}$. A set $\mathfrak{X}^{(1)}$ is compact on a set $\mathfrak{X}^{(0)}$ in case $\mathfrak{X}^{(1)}$ is included in $\mathfrak{X}^{(0)}$, and every infinite sequence of subsets of $\mathfrak{X}^{(1)}$ has an accumulation point in $\mathfrak{X}^{(0)}$. $\uparrow$ This does not exclude the possibility that the sets $\mathfrak{X}^{(1)}$ and $\mathfrak{X}^{(0)}$ may contain only a finite number of points. A set $\mathfrak{X}^{(1)}$ compact on itself is called self-compact.

A neighborhood $\left(\mathfrak{X}^{(0)}\right)_{a}$ of a set $\mathfrak{X}^{(0)}$ of points consists of the sum of the neighborhoods $\left(x_{0}\right)_{a}$ of all points $x_{0}$ of $\mathfrak{X}^{(0)}$.

LEMMA 5.1. A set $\mathfrak{X}^{(1)}$, compact on $\mathfrak{X}$, is bounded. $\ddagger$

LEMma 5.2. A set $\mathfrak{X}^{(1)}$, which is compact on a region $\mathfrak{X}_{0}$, has a neighborhood $\left(\mathfrak{X}^{(1)}\right)_{a}$ contained in $\mathfrak{X}_{0}$.

These two lemmas are verified by the usual indirect proof.

Lemma 5.3. If $\mathfrak{X}$ is a composite space $(\mathfrak{U}, \mathfrak{B})$, and if the set $\mathfrak{X}^{(1)}$ is compact on the set $\mathfrak{X}^{(0)}$, then the projection $\mathfrak{u}^{(1)}$ of $\mathfrak{X}^{(1)}$ on the space $\mathfrak{u}$ is compact on the projection $\mathfrak{u}^{(0)}$ of $\mathfrak{X}^{(0)}$.

* The principal theorem of this sub-section is due to T. H. Hildebrandt.

$\dagger$ With the definition of compactness phrased in this way, it is possible to avoid any use of the postulate of Zermelo. Cf. Cipolla, Atti della Accademia Gioenia in Catania, vol. 6 (1913), Memoir V, Sul postulato di Zermelo e la teoria dei limiti delle funzioni.

‡ Cf. Fréchet, Rendiconti del Circolo Matematico di Palermo, vol. 22 (1906), p. 22. 
Let $\mathfrak{X}^{(1)}$ be a set contained in the set $\mathfrak{X}^{(0)}$, and let $F$ be a function on $\mathfrak{X}^{(0)} \mathfrak{B}$ to a metric space $\mathfrak{Z}$. Then we shall say that $F$ is continuous on $\mathfrak{X}^{(0)}$ uniformly $\left(\mathfrak{X}^{(1)} \mathfrak{B} ; \sigma\right)$ in case $F$ is continuous on $\mathfrak{X}^{(0)}$ uniformly $(\mathfrak{B} ; \sigma)$, and for every positive $\epsilon$ there exists a positive $\delta$ such that, for every $x_{1}$ in $\mathfrak{X}^{(1)}$ and every $x_{0}$ in $\mathfrak{X}^{(0)}$ satisfying $\left\|x_{0}, x_{1}\right\|<\delta$, and for every $p$, we have $\| F\left(x_{0}, p\right)$, $F\left(x_{1}, p\right) \| \leqq \epsilon|\sigma(p)|$.

We say that a function $F$ on $\mathfrak{X}^{(0)} \mathfrak{P}$ to a linear metric space $\mathfrak{Z}$ is bounded $(\mathfrak{B} ; \sigma)$ in case for each $x$ of $\mathfrak{X}^{(0)}$ there exists a constant $M_{x}$ such that $\|F(x, p)\| \leqq M_{x}|\sigma(p)|$ for every $p$. We obtain the definition of modularity as a special case of this in case a component of $\mathfrak{B}$ is a linear metric space $\mathbb{Z}$, and $\|z\|$ is a factor of $\sigma$.

LeMma 5.4. Let the set $\mathfrak{X}^{(1)}$ be compact on the set $\mathfrak{X}^{(0)}$ of the metric space $\mathfrak{X}$, and let the function $F$ on $\mathfrak{X}^{(0)} \mathfrak{B}$ to the metric space $\mathfrak{Y}$ be continuous on $\mathfrak{X}^{(0)}$ uniformly $(\mathfrak{B} ; \sigma)$. Then $F$ is continuous on $\mathfrak{X}^{(0)}$ uniformly $\left(\mathfrak{X}^{(1)} \mathfrak{B} ; \sigma\right)$.

LEMMA 5.5. Let the set $\mathfrak{X}^{(1)}$ be compact on the set $\mathfrak{X}^{(0)}$ of the metric space $\mathfrak{X}$, and let $\mathfrak{\eta}$ be a linear metric space. Let the function $F$ on $\mathfrak{X}^{(0)} \mathfrak{B}$ to $\mathfrak{\eta}$ be continuous on $\mathfrak{X}^{(0)}$ uniformly $(\mathfrak{B} ; \sigma)$, and bounded $(\mathfrak{B} ; \sigma)$. Then $F$ is bounded $\left(\mathfrak{X}^{(1)} \mathfrak{B} ; \sigma\right)$.

The usual indirect proofs suffice for both Lemma 5.4 and Lemma 5.5.

In case the spaces $\mathfrak{X}$ and $\mathfrak{Y}$ are linear, we say that the function $F$ on $\mathfrak{X}_{0} \mathfrak{B}$ to $\mathfrak{Y}$ is of class $\mathfrak{S}^{\prime}$ on $\mathfrak{X}_{0}$ uniformly $\left(\mathfrak{X}^{(1)} \mathfrak{B} ; \sigma\right)$ under the following conditions: (0) $F$ is of class $\mathbb{E}^{\prime}$ on $\mathfrak{X}_{0}$ uniformly $(\mathfrak{P} ; \sigma) ;(1) d F$ is continuous on $\mathfrak{X}_{0}$ uniformly $\left(\mathfrak{X}^{(1)} \mathfrak{B X} ; \sigma\|d x\|\right) ;(2) d F$ is modular on $\mathfrak{X}$ uniformly $\left(\mathfrak{X}^{(1)} \mathfrak{B}\right.$; $\sigma)$; (3) for every positive $\epsilon$ there is a positive $\delta$ such that, whenever the points $x_{1}$ of $\mathfrak{X}^{(1)}$ and $x_{0}$ of $\mathfrak{X}_{0}$ satisfy $\left\|x_{0}-x_{1}\right\|<\delta$, then

$$
\begin{aligned}
\| F\left(x_{0}, p\right)-F\left(x_{1}, p\right)-d F\left(x_{1}, p\right. & \left.; x_{0}-x_{1}\right) \| \\
& =\left\|R\left(x_{0}, x_{1}, p\right)\right\|\left\|x_{0}-x_{1}\right\| \leqq \epsilon|\sigma(p)|\left\|x_{0}-x_{1}\right\| .
\end{aligned}
$$

This special uniformity is extended inductively in the usual manner to the class $\mathbb{E}^{(n)}$.

TheOREM II. Let the set $\mathfrak{X}^{(1)}$ be compact on the region $\mathfrak{X}_{0}$ of the linear metric space $\mathfrak{X}$, and suppose that the function $F$ on $\mathfrak{X}_{0} \mathfrak{B}$ to the linear metric space $\mathfrak{V}$ is of class $\mathfrak{S}^{(n)}$ on $\mathfrak{X}_{0}$ uniformly $(\mathfrak{B} ; \sigma)$. Then $F$ is of class $\mathfrak{E}^{(n)}$ on $\mathfrak{X}_{0}$ uniformly $\left(\mathfrak{X}^{(1)} \mathfrak{B} ; \sigma\right)$.

Consider first the case $n=1$. Properties (1) and (2) in the definition above follow from Lemmas 5.4 and 5.5. For property (3), we could proceed directly if the region $\mathfrak{X}_{0}$ were convex, but we shall use the indirect proof which covers 
all cases. Suppose then that the last property does not hold. Then there exists a positive number $\epsilon$ such that for every positive integer $m$ there exist a set $\mathfrak{X}^{(0 m)}$ of points $x_{0 m}$ in $\mathfrak{X}_{0}$, a set $\mathfrak{X}^{(1 m)}$ of points $x_{1 m}$ in $\mathfrak{X}^{(1)}$, and a set $\mathfrak{B}^{(m)}$ of elements $p_{m}$ of the range $\mathfrak{B}$, such that

$$
\left\|x_{0 m}-x_{1 m}\right\| \leqq \frac{1}{m}, \quad\left\|R\left(x_{0 m}, x_{1 m}, p_{m}\right)\right\|>\epsilon\left|\sigma\left(p_{m}\right)\right| .
$$

The sequence of sets $\left\{\mathfrak{X}^{(1 m)}\right\}$ has an accumulation point $x_{0}$ which is in $\mathfrak{X}_{0}$, and hence there is a neighborhood $\left(x_{0}\right)_{a}$ contained in $\mathfrak{X}_{0}$. Then for every integer $m^{\prime}$ there is always an $m>m^{\prime}$ such that there are points $x_{0 m}$ and $x_{1 m}$ of the sets $\mathfrak{X}^{(0 m)}$ and $\mathfrak{X}^{(1 m)}$ respectively, which are in $\left(x_{0}\right)_{a}$. The neighborhood $\left(x_{0}\right)_{a}$ is a convex region, so that we can apply Taylor's theorem* to obtain (omitting $p$ )

$$
\begin{array}{r}
R\left(x_{0 m}, x_{1 m}\right)\left\|x_{0 m}-x_{1 m}\right\|=\int_{0}^{1}\left\{d F\left(x_{1 m}+\left(x_{0 m}-x_{1 m}\right) r, x_{0 m}-x_{1 m}\right)\right. \\
\left.-d F\left(x_{1 m}, x_{0 m}-x_{1 m}\right)\right\} d r .
\end{array}
$$

We have already shown that $d F$ is continuous on $\mathfrak{X}_{0}$ uniformly $\left(\mathfrak{X}^{(1)} \mathfrak{B X}\right.$; $\sigma\|d x\|)$, so that we have

$$
\left\|R\left(x_{0 m}, x_{1 m}\right)\right\| \leqq \epsilon|\sigma|
$$

whenever $\left\|x_{0 m}-x_{1 m}\right\|$ is sufficiently small, i.e., when $m^{\prime}$ is sufficiently large. This is the desired contradiction.

The proof of the theorem is completed by an obvious induction.

In the case when the set $\mathfrak{X}^{(1)}$ contains only a finite number of points, the theorem is obvious and trivial.

6. Equivalence of our definition with the classical definition of the class $\mathfrak{E}^{(n)}$. Consider an $m$-dimensional euclidean space $\mathfrak{X}$, with the usual definition of distance. Let $F$ be a single-real-valued function on a region $\mathfrak{X}_{0}$ of $\mathfrak{X}$ i.e., $F$ is on $\mathfrak{X}_{0}$ to $\Re$. Then the function $F$ is of class $\mathfrak{E}^{(n)}$ on $\mathfrak{X}_{0}$ according to Bolza $\dagger$ in case $F$ has partial derivatives up to and including those of the $n$th order, all of which are continuous on $\mathfrak{X}_{0}$. The equivalence of this with our definition is stated in the following

THEOREM III. If the function $F$ is of class $\mathfrak{G}^{(n)}$ on $\mathfrak{X}_{0}$ according to the definition of Paper $\mathrm{I}$, then $F$ is of class $\mathcal{E}^{(n)}$ on $\mathfrak{X}_{0}$ according to Bolza, and conversely.

* See Paper II.

† See Vorlesungen über Variationsrechnung, p. 13. 
It is convenient to introduce a general range $\mathfrak{B}$ with scale function $\sigma$, and to prove the more general

THEOREM III'. If the function $F$ on $\mathfrak{X}_{0} \Re$ to $\Re$ is of class $\mathfrak{S}^{(n)}$ on $\mathfrak{X}_{0}$ uniformly $(\Re ; \sigma)$, then $F$ has partial derivatives up to and including those of the nth order, all of which are continuous on $\mathfrak{X}_{0}$ uniformly $(\mathfrak{P} ; \sigma)$, and bounded $(\mathfrak{B} ; \sigma)$ for each $x$ of $\mathfrak{X}_{0}$. The converse is also true.

Note that the function $F$ itself is not necessarily bounded $(\mathfrak{B} ; \sigma)$. Although the range $\mathfrak{P}$ is essential in the induction, we can omit writing the variable $p$ for the most part, as it enters "homogeneously."

We shall denote the coördinates of a point $x$ by $x^{i}(i=1, \cdots, m)$, and the unit points on the various axes will be denoted by $u_{i}(i=1, \cdots, m)$, i.e., $u_{i}^{j}=0$ for $j \neq i$, and $=1$ for $j=i$.

Consider the theorem for $n=1$. The $m$ functions $d F\left(x, u_{i}\right)$ are evidently the partial derivatives of $F$, and have the required properties.

To complete the proof by induction, suppose that $F$ is of class $C^{(k+1)}$ on $\mathfrak{X}_{0}$. Then if the theorem is true for $n=k$, each function $d F\left(x, u_{i}\right)$ has partial derivatives of the $k$ th order. Hence $F$ has partial derivatives of the $(k+1)$ st order.

To prove the converse, we define the function $d F$ on $\mathfrak{X}_{0} \mathfrak{X}$ to $\Re$ by the equation

$$
d F(x, d x)=\sum_{1}^{m} \frac{\partial F(x)}{\partial x^{i}} d x^{i} .
$$

The continuity and linearity of $d F$ are then obvious. For the third condition we have, by the classical theorem of the mean,

$$
R\left(x_{1}, x\right)\left\|x_{1}-x\right\|=\sum_{1}^{m}\left(\frac{\partial F\left(x+\theta\left(x_{1}-x\right)\right)}{\partial x^{i}}-\frac{\partial F(x)}{\partial x^{i}}\right)\left(x_{1}^{i}-x^{i}\right)
$$

$$
(0<\theta<1) \text {, }
$$

whence $R\left(x_{1}, x\right)$ approaches zero with $\left\|x_{1}-x\right\|$ uniformly $(\mathfrak{B} ; \sigma)$.

To complete the proof by induction, we assume that the theorem is true for $n=k$, and that $F$ has derivatives of order $k+1$ with the required properties. Then it is readily verified that the function $d F$ has partial derivatives up to the $k$ th order which are continuous on $\mathfrak{X}_{0}$ uniformly ( $\mathfrak{B X}$; $\sigma\|d x\|)$ and bounded $(\mathfrak{B X} ; \sigma\|d x\|)$. Hence $d F$ is of class $\mathfrak{S}^{(k)}$ on $\mathfrak{X}_{0}$ uniformly $(\mathfrak{B X} ; \sigma\|d x\|)$, so that $F$ is of class $\left(^{(k+1)}\right.$ as required.

7. The lemmas of this paragraph will occasionally be of use in dealing with differentials of order higher than the first. All the spaces $\mathfrak{X}, \mathfrak{Z}, \mathbb{Z}$ considered are assumed to be linear metric spaces. 
There will be occasion to consider differentiation of functions $F(p, x)$ on $\mathfrak{B X}_{p}$ to $\mathfrak{Y}$, where the range $\mathfrak{X}_{p}$ of the argument $x$ depends on the argument $p$. In such cases it is obviously suitable to write the range $\mathfrak{P}$ first. The range $\mathfrak{B}$ may of course be composite, and $\mathfrak{X}_{p}$ may then actually depend on only one component of $\mathfrak{B}$. We say that a function $F$ on $\mathfrak{B X} \mathfrak{X}_{\mathfrak{p}}$ to $\mathfrak{Y}$ is continuous on $\mathfrak{X}_{p}$ uniformly $\left(\mathfrak{B X} \mathfrak{X}_{p} ; \sigma\right)$ in case for every positive $\epsilon$ there is a positive $\delta$ such that, for every $p$ and every $x_{1}$ and $x_{2}$ in $\mathfrak{X}_{p}$ having $\left\|x_{1}-x_{2}\right\| \leqq \delta$ we have

$$
\left\|F\left(p, x_{1}\right)-F\left(p, x_{2}\right)\right\| \leqq \epsilon|\sigma(p)| .
$$

This indicates how we define the class $\mathbb{S}^{\prime}$ on $\mathfrak{X}_{p}$ uniformly $\left(\mathfrak{B X} \mathfrak{X}_{p} ; \sigma\right)$.

LEMma 7.1. Let the function $F$ on $\mathfrak{P X}_{p} \mathfrak{Z}$ to $\mathbb{Z}$ be distributive on $\mathfrak{Z}$ for each $p$ and $x$. Let $b$ be a positive constant, and let $\mathfrak{V}^{(b)}$ denote the set of points $y$ such that $\|y\|=b$. Then if $F$ is of class $\mathfrak{S}^{\prime}$ on $\mathfrak{X}_{p}$ uniformly $\left(\mathfrak{B X}_{p} \mathfrak{V}^{(b)} ; \sigma\right), F$ is also of class $\mathbb{E}^{\prime}$ on $\mathfrak{X}_{p}$ uniformly $\left(\mathfrak{B X}_{p} \mathfrak{Y} ; \sigma\|y\|\right)$, and conversely. Moreover the differential $d_{x} F$ is linear on $\mathfrak{Y}$ uniformly $\left(\mathfrak{B} \mathfrak{X}_{p} \mathfrak{X} ; \sigma\|d x\|\right)$.

The lemma can readily be extended by induction to the case where $F$ is of class $\mathcal{G}^{(n)}$, and there are $m$ spaces entering after the fashion of the space Y. But we shall not need anything more general than the lemma stated. The proof is as follows.

We first define a function $G$ on $\mathfrak{B X}_{p} \mathfrak{Y} \mathfrak{X}$ to $\mathbb{Z}$ by the equations

$$
\begin{array}{rlrl}
G(p, x, y ; d x) & =d_{x} F\left(p, x, y b^{\prime} ; d x\right) \frac{1}{b^{\prime}}, & \\
b^{\prime} & =\frac{b}{\|y\|} \quad\left(y \neq y_{*}\right), \\
G\left(p, x, y_{*} ; d x\right) & =z_{*} .
\end{array}
$$

Then it is easy to verify that $G$ is the differential $d_{x} F$ of $F$, and satisfies the conditions for $F$ to be of class $\mathbb{E}^{\prime}$ on $\mathfrak{X}_{p}$ uniformly $\left(\mathfrak{B X}_{p} \mathfrak{Y} ; \sigma\|y\|\right)$. The converse part of the lemma is obvious.

By use of Lemma 11.1 of Paper I, we can show that $d_{x} F$ is distributive on Y). The modularity of $d_{x} F$ on $\mathfrak{Y}$ uniformly $(\mathfrak{B X} \mathfrak{X} ; \sigma\|d x\|)$ is equivalent to the modularity of $d_{x} F$ on $\mathfrak{X}$ uniformly $\left(\mathfrak{B X} \mathfrak{X}_{p} \mathfrak{Y} ; \sigma\|y\|\right)$.

LEMMA 7.2. Let the function $F$ on $\mathfrak{B X}_{p} \mathfrak{Y}$ to $\mathbb{3}$ be linear on $\mathfrak{Y}$ uniformly $\left(\mathfrak{P X}_{\mathfrak{p}} ; \sigma\right)$, and let $b$ be a positive number. Then if $F$ is of class $\mathbb{E}^{(n)}$ on $\mathfrak{X}_{p}$ uniformly $\left(\mathfrak{B X}_{p} \mathfrak{Y} ; \sigma\|y\|\right), F$ is also of class $\mathfrak{S}^{(n)}$ on the composite region $\left(\mathfrak{X}_{p}\left(y_{*}\right) b\right)$ uniformly $\left(\mathfrak{B X}_{p}\left(y_{*}\right)_{b} ; \sigma\right)$, and conversely.

This lemma could also be extended to the case where there are $m$ spaces entering in the fashion of the space $\mathfrak{Y}$. 
To prove the lemma as it stands, we consider first the case $n=1$. Denote the region $\left(\mathfrak{X}_{p},\left(y_{*}\right)_{b}\right)$ by $\mathfrak{W}_{p}$. The differential $d_{x} F$, and the differential $d_{y} F$ defined by

$$
d_{y} F(p, x, y ; d y)=F(p, x, d y)
$$

evidently satisfy the uniformity requirements of Lemma 13.2 of Paper I, so that we can say that $F$ is of class $\mathfrak{C}^{\prime}$ on $\mathfrak{W}_{p}$ uniformly $\left(\mathfrak{B W}_{p} ; \sigma\right)$.

To complete the proof by induction, we assume that the lemma is true for $n=k$, and that $F$ is of class $\mathfrak{C}^{(k+1)}$ on $\mathfrak{X}_{p}$ uniformly $\left(\mathfrak{B X} \mathfrak{X}_{p} \mathfrak{Y} ; \sigma\|y\|\right)$. Then $d_{x} F$ is of class $\mathfrak{S}^{(k)}$ on $\mathfrak{X}_{p}$ uniformly $(\mathfrak{B X} \mathfrak{X} \mathfrak{Y} \mathfrak{X} ;\|y\|\|d x\|)$. Also $d_{x} F$ is linear on $\mathfrak{Y}$ uniformly $(\mathfrak{P X} \mathfrak{X} ; \sigma\|d x\|)$. Hence by the present lemma for $n=k$, $d_{x} F$ is of class $\mathfrak{C}^{(k)}$ on $\mathfrak{W}_{p}$ uniformly $\left(\mathfrak{P W}_{p} \mathfrak{X} ; \sigma\|d x\|\right)$. By Lemma 14.1 of Paper I, $d_{y} F$ (as defined by equation (7.1)), is of class $\mathfrak{S}^{(k)}$ on $\mathfrak{X}_{p}$ uniformly $\left(\mathfrak{B} \mathfrak{X}_{p} \mathfrak{Y} ; \sigma\|d y\|\right)$, and since $d_{y} F$ is independent of $y$, it is also of class $\mathfrak{S}^{(k)}$

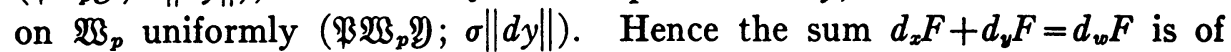
class $\mathfrak{C}^{(k)}$ on $\mathfrak{W}_{p}$ uniformiy $\left(\mathfrak{B W}_{p} \mathfrak{W} ; \sigma\|d w\|\right)$, so that $F$ is of class $\mathfrak{( S}^{(k+1)}$ on $\mathfrak{W}_{p}$ uniformly $\left(\mathfrak{P}_{p} ; \sigma\right)$. This completes the induction.

To prove the converse for $n=1$, we have from Lemma 13.2 of Paper I that $F$ is of class $\mathfrak{S}^{\prime}$ on $\mathfrak{X}_{p}$ uniformly $\left(\mathfrak{B} \mathfrak{X}_{p}\left(y_{*}\right)_{b} ; \sigma\right)$, and hence uniformly $\left(\mathfrak{B X} \mathfrak{Y}^{\left(b^{\prime}\right)} ; \sigma\right)$, where $b^{\prime}<b$. Then Lemma 7.1 gives the desired conclusion.

To complete the proof by induction, we make the usual assumptions. Then $d_{x} F$ is of class $\mathfrak{C}^{(k)}$ on $\mathfrak{B}_{p}$ uniformly $\left(\mathfrak{B M} \mathfrak{W}_{p} \mathfrak{X} ; \sigma\|d x\|\right)$, and linear on $\mathfrak{V}$ uniformly $(\mathfrak{B X} \mathfrak{X} ; \sigma\|d x\|)$. Hence by the lemma for $n=k, d_{x} F$ is of class $\mathcal{C}^{(k)}$ on $\mathfrak{X}_{p}$ uniformly $\left(\mathfrak{B X} \mathfrak{X}_{p} \mathfrak{Y} \mathfrak{X} ; \sigma\|y\|\|d x\|\right)$, so that by definition, $F$ is of class $\mathfrak{C}^{(k+1)}$ on $\mathfrak{X}_{p}$ uniformly $\left(\mathfrak{B} \mathfrak{X}_{p} \mathfrak{Y} ; \sigma\|y\|\right)$.

III. ThE SOlution OF THE EQUATION $G(x, y)=y_{*}$ ON SUBSPACES

8. Nature of the subspaces considered. We shall be considering linear metric subspaces $\mathfrak{X}^{\prime}$ of linear metric spaces $\mathfrak{X}$, the definition of the operations \|\|$, \oplus, \odot$ being unchanged in the subspace. As a special case we may have $\mathfrak{X}^{\prime}=\mathfrak{X}$. In particular, following out the practice of Parts IV and $V$ of Paper I, we shall denote by $\mathfrak{Y}^{\prime}$ a complete linear metric subspace of a complete linear metric space $\mathfrak{Y}$. If we set $\mathfrak{W}=(\mathfrak{X}, \mathfrak{Z}), \mathfrak{W}=\left(\mathfrak{X}^{\prime}, \mathfrak{Y}^{\prime}\right)$, then $\mathfrak{W}^{\prime}$ is a linear metric subspace of $\mathfrak{W}$.

Corresponding to a region $\mathfrak{X}_{0}$ of $\mathfrak{X}$ we shall denote by $\left(\mathfrak{X}_{0}\right)^{\prime}$ the set of points common to $\mathfrak{X}_{0}$ and a subspace $\mathfrak{X}^{\prime}$. If $F$ is a function on $\mathfrak{X}_{0}$ to $\mathfrak{Z}$ such that, when $x$ is in $\left(\mathfrak{X}_{0}\right)^{\prime}, F(x)$ is in the subspace $\mathfrak{Y}^{\prime}$ of $\mathfrak{Y}$, we shall say that $F$ as on $\left(\mathfrak{X}_{0}\right)^{\prime}$ is to $\mathfrak{Y}^{\prime}$. We propose to determine the nature of the solution of the equation $G(x, y)=y_{*}$, when the initial solution $\left(x_{0}, y_{0}\right)$ is in $\mathfrak{W}^{\prime}$, and the function $G$ as on $\left(\mathfrak{W}_{0}\right)^{\prime}$ is to $\mathfrak{Y}^{\prime}$. 
9. Limits, regions, and differentials in subspaces. We prove the following lemmas.

LEMMA 9.1. If a sequence $\left\{y_{n}^{\prime}\right\}$ of elements of $\mathfrak{Y}^{\prime}$ has a limit $y$ in $\mathfrak{Y}$, then $y$ is in $\mathfrak{Y}^{\prime}$.

This follows from the completeness of $\mathfrak{Y}^{\prime}$, and the uniqueness of limit in $\mathfrak{Y}$.

LEMMA 9.2. If $\mathfrak{X}_{0}$ is a region of $\mathfrak{X}$ containing points of $\mathfrak{X}^{\prime}$, then the set $\left(\mathfrak{X}_{0}\right)^{\prime}$ is a region in the space $\mathfrak{X}^{\prime}$.

This follows readily from the definition of region.

Lemma 9.3. Let $F$ be a function on $\mathfrak{X}_{0}$ to $\mathfrak{Y}$, having a differential $d F$ at a point $x_{0}^{\prime}$ of the region $\left(\mathfrak{X}_{0}\right)^{\prime}$, and suppose that $F$ as on $\left(\mathfrak{X}_{0}\right)^{\prime}$ is to $\mathfrak{Y}^{\prime}$. Then the differential $d F$ as on $\mathfrak{X}^{\prime}$ is to $\mathfrak{Y}^{\prime}$.

This follows from Lemma 11.1 of Paper I, and Lemma 9.1.

10. The theorems of this paragraph are corollaries or additions to Theorems IV and V of Paper I. The latter, we shall call Theorems IVI, $\mathrm{V}^{\mathrm{I}}$ respectively. For convenience we retain here all the notations, hypotheses, and conclusions of Theorems IV ${ }^{\mathrm{I}}$ and $\mathrm{V}^{\mathrm{I}}$, and state only the additional hypotheses and conclusions.

TheOREM IV. Suppose that the initial solution $\left(x_{0}, y_{0}\right)$ is in $\mathfrak{B}^{\prime}$, and that the function $G$ as on $\left(\mathfrak{B}_{0}\right)^{\prime}$ is to $\mathfrak{Y}^{\prime}$. Suppose also that the reciprocal function $L_{0}$ as on $\mathfrak{Y}^{\prime}$ is to $\mathfrak{Y}^{\prime}$. Then the solution $Y$ as on $\left(\left(x_{0}\right)_{b}\right)^{\prime}$ is to $\left(\left(y_{0}\right)_{a}\right)^{\prime}$, and the reciprocal $L$ of $d_{y} G(x, Y(x) ; d y)$ as on $\left(\left(x_{0}\right)_{b}\right)^{\prime} Y^{\prime}$ is to $\mathfrak{Y}^{\prime}$.

By Lemma 9.3, the function $G$, as on $\left(\mathfrak{B}_{0}\right)^{\prime}$ to $\mathfrak{Y}^{\prime}$, is of class $\mathbb{C}^{\prime}$ on $\left(\mathfrak{B}_{0}\right)^{\prime}$. Thus all the hypotheses of Theorem IV ${ }^{\mathrm{I}}$ (for $n=1$, at least) are satisfied with $\mathfrak{X}$ replaced by $\mathfrak{X}^{\prime}$ and $\mathfrak{Y}$ by $\mathfrak{Y}^{\prime}$, so that there exist positive constants $a^{\prime}$ and $b^{\prime}$ and a unique function $Y^{\prime}$ on $\left(\left(x_{0}\right)_{b^{\prime}}\right)^{\prime}$ to $\left(\left(y_{0}\right)_{a^{\prime}}\right)^{\prime}$ satisfying the conclusions $C_{1}$ to $C_{4}$ of Theorem IVI. By inspection of the proofs of Theorem $\mathrm{IV}^{\mathrm{I}}$ and Lemma 16.2 ${ }^{\mathrm{I}}$, it is seen that the constants $a^{\prime}$ and $b^{\prime}$ are restricted only by the continuity and modularity properties of $G, d_{y} G$, and $L_{0}$, so that we may take $a^{\prime}=a, b^{\prime}=b$. Then by the uniqueness of the solution, we have $Y^{\prime}=Y$ on $\left(\left(x_{0}\right)_{b}\right)^{\prime}$.

THEOREM V. In addition to the assumptions of Theorem IV, suppose that the subspace $\mathfrak{X}^{\prime}$ is identical with the space $\mathfrak{X}$. Then the sheet $\mathfrak{B}^{(0)}$ of solutions, defined in Theorem $\mathrm{V}^{\mathrm{I}}$, lies wholly in the space $\mathfrak{B}^{\prime}$. 
To prove this statement, let $w_{1}$ be a point of the sheet $\mathfrak{W B}^{(0)}$, and let $w_{1}$ be connected to the initial point $w_{0}=\left(x_{0}, y_{0}\right)$ by a continuous function $W$ on the interval (01) to $\mathfrak{B}^{(0)}$. Then by the definition of a sheet, and Theorem IV, there exist points $r_{2}$ in the interval (01) such that, for $0 \leqq r \leqq r_{2}$ we have (1) $W(r)$ is in $\mathfrak{B}^{\prime}$, and (2) the reciprocal $L$ of $d_{y} G$ at $W(r)$ has the property that $L$ as on $\mathfrak{y}^{\prime}$ is to $\mathfrak{y}^{\prime}$. Let $r_{\mathbf{3}}$ be the upper bound of such points $r_{2}$. Then by the continuity of the function $W$ and of the reciprocal of $d_{y} G$, and by Lemma 9.1, the conditions (1) and (2) are satisfied for $r=r_{3}$. If $r_{3} \neq 1$, there is by Theorem IV a neighborhood of $r_{3}$ on which the same conditions hold. This is a contradiction, so that the point $w_{1}$ is actually in $\mathfrak{B}^{\prime}$.

11. Extent of the maximal sheet of solutions in a subspace. When we consider the equation $G(x, y)=y_{*}$ in a subspace $\mathfrak{Y}^{\prime}$ of $\mathfrak{\eta}$, the maximal sheet of solutions may extend farther in the subspace than in the main space. This is shown by such a simple example as the following. Le the space $\mathfrak{X}$ be a one-dimensional space, and the space $\mathfrak{V}$ a two-dimensional sace, and denote the coördinate of a point $x$ simply by $x$, and the coörd ites of a point $y$ by $y_{1}$ and $y_{2}$. Let $\mathfrak{Y}^{\prime}$ ' be the one-dimensional subspace of $\mathfrak{\eta}$. ' which $y_{1}=y_{2}$. We take for the function $G$ the one whose coördinates are

$$
\begin{aligned}
& (3-x) y_{1}+(x-1) y_{2}-x, \\
& (1+x) y_{1}+(1-x) y_{2}-x .
\end{aligned}
$$

Then $G$ as on $\mathfrak{X \mathfrak { Y } ^ { \prime }}$ is to $\mathfrak{Y}^{\prime}$. Also for each $x \neq 1$, the differential $d_{y} G$ has a reciprocal $K$ on $\mathfrak{Y}$ to $\mathfrak{Y}$, and $K$ as on $\mathfrak{Y}^{\prime}$ is to $\mathfrak{Y}^{\prime}$. Now a solution of the equation $G=y_{*}$ has the form $y_{1}=y_{2}=x / 2$, valid for all values of $x$, and unique except for $x=1$. But for $x=1, d_{y} G$ has no reciprocal in the space $\mathfrak{Y}$, so that a maximal sheet $\mathfrak{B}^{(0)}$ of solutions in the space $\mathfrak{V}$ would have the point $x=1, y_{1}=y_{2}$ $=1 / 2$, for a boundary point. But even at $x=1, d_{y} G$ has a reciprocal in the space $\mathfrak{Y}^{\prime}$, so that the maximal sheet $\mathfrak{B}^{(0)}$ in the space $\mathfrak{Y}^{\prime}$ can have no boundary point.

\section{A SPECIAL MODE OF DEPENDENCE OF THE FUNCTION $G$ UPON $x$ AND $y$, AND AN EXTENDED IMPLICIT FUNCTION THEOREM}

12. Nature of the spaces $\mathfrak{X}$ and $\mathfrak{Y}$, and special notations. In this section we shall be considering spaces $\mathfrak{X}$ and $\mathfrak{Z}$ of type $B$. More specifically let $\mathfrak{U}$ be a linear metric space and $\mathfrak{B}$ be a complete linear metric space. Let $\mathfrak{B}$ be a general range, and let the scale function $\sigma$ be identically equal to unity. Then let $\mathfrak{X}$ be the space of type $B$ consisting of functions $x$ on $\mathfrak{B}$ to $\mathfrak{u}$, and let $\mathfrak{V}$ be the space of type $B$ consisting of functions $y$ on $\mathfrak{B}$ to $\mathfrak{B}$. The space $\mathfrak{V}$ therefore is complete. It is not necessary to restrict the spaces $\mathfrak{X}$ and $\mathfrak{V}$ so strongly for the first lemmas, but the possible extensions are easily noted. 
Corresponding to a region $\mathfrak{X}_{0}$ of a space $\mathfrak{X}$, there will be a system of regions $\mathfrak{u}_{p}$ in the space $\mathfrak{u}$, where $\mathfrak{u}_{p}$ consists of all those points $u$ for which there is a function $x$ in $\mathfrak{X}_{0}$ having $x(p)=u$. It is readily verified that each $\mathfrak{u}_{p}$ so defined actually is a region. Conversely, corresponding to an arbitrary system of regions $\mathfrak{U}_{p}$ there is usually a region $\mathfrak{X}_{0}$ to which the system of regions $\mathfrak{u}_{p}$ corresponds as above. But such a region $\mathfrak{X}_{0}$ will not always be uniquely determined by this condition. If a region $\mathfrak{X}_{0}$ exists corresponding to a system of regions $\mathfrak{u}_{p}$, the most inclusive such region consists of all points $x$ of the space $\mathfrak{X}$ for which there is a positive constant $a_{x}$ such that the neighborhood $(x(p))_{a_{x}}$ is in $\mathfrak{u}_{p}$, for every $p$. To avoid questions of existence we shall throughout this section assume that the region $\mathfrak{X}_{0}$ is given and that the system of regions $\mathfrak{U}_{p}$ corresponds to it. We shall also retain the notation $F\left(p^{\prime} \mid p\right)$ introduced in $\S 3$.

13. Preliminary theorems on differentials and reciprocals. We prove the following lemmas.

LEMMA 13.1. Let $G$ be a function on $\mathfrak{X}_{0} \mathfrak{P}^{\prime}$ to $\mathfrak{V}$, of class $\mathcal{E}^{(n)}$ on $\mathfrak{X}_{0}$ uniformly $\left(\mathfrak{P}^{\prime} ; \sigma^{\prime}\right)$, and suppose that $G\left(x, p^{\prime} \mid p\right)$ depends on the function $x$ only at $p$, for every $p$ and $p^{\prime}$. Then the function $H$ on $\mathfrak{P u}_{p} \mathfrak{P}^{\prime}$ to $\mathfrak{B}$ defined by

$$
G\left(x, p^{\prime} \mid p\right)=H\left(p, u, p^{\prime}\right), \quad u=x(p),
$$

is of class $\mathfrak{E}^{(n)}$ on each region $\mathfrak{U}_{p}$ uniformly $\left(\mathfrak{B}^{\prime} ; \sigma^{\prime}\right)$.

The ranges of uniformity in this lemma may be modified in special cases. E.g., if the region $\mathfrak{u}_{p}$ is the same for every $p$, then $\mathfrak{B}$ may be inserted in the range of uniformity in the conclusion. If the region $\mathfrak{X}_{0}$ is a neighborhood $\left(x_{0}\right)_{a}$, then $\mathfrak{X}_{0}$ and $\mathfrak{B} \mathfrak{u}_{p}$ may be inserted in the ranges of uniformity of the hypothesis and conclusion respectively. The outline of the proof is unaltered.

To prove the lemma we must first show that the differential $d_{x} G$ has the property that $d_{x} G\left(x, p^{\prime} ; d x \mid p\right)$ depends on $x$ and $d x$ only at $p$. Let $x_{1}(p)=x_{2}(p)$, $d_{1} x(p)=d_{2} x(p)$, for a certain $p$. Then from the assumed property of the function $G$, Lemma 11.1 of Paper I, and the uniqueness of limits, we find that $d_{x} G\left(x_{1}, p^{\prime} ; d_{1} x \mid p\right)=d_{x} G\left(x_{2}, p^{\prime} ; d_{2} x \mid p\right)$. Hence we can define the function $d_{\mathfrak{u}} H$ on $\mathfrak{B} \mathfrak{u}_{p} \mathfrak{P}^{\prime} \mathfrak{U}$ to $\mathfrak{B}$ by

$$
d_{x} G\left(x, p^{\prime} ; d x \mid p\right)=d_{u} H\left(p, u, p^{\prime} ; d u\right), \quad u=x(p), \quad d u=d x(p) .
$$

To prove the continuity of $d_{u} H$, let

$$
\begin{aligned}
u_{2} & =x_{2}\left(p_{0}\right) \\
x_{1}(p) & =x_{2}(p)+u_{1}-u_{2} \text { for every } p, \\
d x(p) & =d u \text { for every } p .
\end{aligned}
$$


We have immediately

$$
\left\|u_{1}-u_{2}\right\|=\left\|x_{1}-x_{2}\right\|, \quad\|d u\|=\|d x\| .
$$

If $u_{2}$ is in $\mathfrak{u}_{p_{0}}, x_{2}$ can be assigned in $\mathfrak{X}_{0}$, and $x_{1}$ is then also in $\mathfrak{X}_{0}$ if $u_{1}$ is sufficiently near $u_{2}$. Then

$$
\begin{aligned}
d_{u} H\left(u_{1}, p_{0}, p^{\prime} ; d u\right)-d_{u} H\left(u_{2}, p_{0}, p^{\prime} ; d u\right) & \\
& =d_{x} G\left(x_{1}, p^{\prime} ; d x \mid p_{0}\right)-d_{x} G\left(x_{2}, p^{\prime} ; d x \mid p_{0}\right) .
\end{aligned}
$$

From this the required continuity of $d_{u} H$ is apparent.

The other two conditions for $H$ to be of class $\mathbb{S}^{\prime}$ are obtained as readily.

We complete the proof by induction. Assume that the lemma is true for $n=k$, and that $G$ is of class $\mathbb{S}^{(k+1)}$. Then $d_{x} G$ is of class $\mathcal{E}^{(k)}$ on $\mathfrak{X}_{0}$ uniformly $\left(\mathfrak{P}^{\prime} \mathfrak{X} ; \sigma^{\prime}\|d x\|\right)$, and $d_{x} G\left(x, p^{\prime} ; d x \mid p\right)$ depends on $x$ only at $p$. If we set $d x(p)$ $=d u$ for all $p$, then the conditions of the lemma are satisfied with $G$ replaced by $d_{x} G, \mathfrak{P}^{\prime}$ replaced by $\mathfrak{P}^{\prime} \mathfrak{U}$, and $n=k$. Hence $d_{u} H$ is of class $\mathfrak{E}^{(k)}$ on each $\mathfrak{U}_{p}$ uniformly $\left(\mathfrak{P}^{\prime} \mathfrak{U} ; \sigma^{\prime}\|d u\|\right)$, so that $H$ is of class $\mathfrak{S}^{(k+1)}$ as required.

The next lemma is a converse of the preceding, but it requires a stronger uniformity.

LEMma 13.2. Consider a region $\mathfrak{X}_{0}$ of the space $\mathfrak{X}$, and the corresponding system of regions $\mathfrak{u}_{p}$ of the space $\mathfrak{u}$. Let $H$ be a function on $\mathfrak{B} \mathfrak{u}_{p} \mathfrak{P}^{\prime}$ to $\mathfrak{B}$, of class $\mathfrak{S}^{(n)}$ on $\mathfrak{U}_{p}$ uniformly $\left(\mathfrak{P} \mathfrak{U}_{p} \mathfrak{P}^{\prime} ; \sigma^{\prime}\right)$, and such that $\left\|H\left(p, x(p), p^{\prime}\right)\right\|$ is bounded on $\mathfrak{P}$ for every $x$ in $\mathfrak{X}_{0}$ and every $p^{\prime}$ of $\mathfrak{P}^{\prime}$. Then the function $G$ on $\mathfrak{X}_{0} \mathfrak{P}^{\prime}$ to $\mathfrak{V}$ defined by

$$
G\left(x, p^{\prime} \mid p\right)=H\left(p, x(p), p^{\prime}\right)
$$

is of class $\mathfrak{S}^{(n)}$ on $\mathfrak{X}_{0}$ uniformly $\left(\mathfrak{X}_{0} \mathfrak{B}^{\prime} ; \sigma^{\prime}\right)$.

Take first the case $n=1$, and define the function $d_{x} G$ on $\mathfrak{X}_{0} \mathfrak{B}^{\prime} \mathfrak{X}$ to $\mathfrak{Y}$ by the equation

$$
d_{x} G\left(x, p^{\prime} ; d x \mid p\right)=d_{u} H\left(p, x(p), p^{\prime} ; d x(p)\right) .
$$

That $d_{x} G$ is to $\mathfrak{Y}$ follows from the uniform modularity of the function $d_{u} H$. The conditions for $G$ to be of class ${ }^{\prime}{ }^{\prime}$ with $d_{x} G$ as its differential are readily verified.

To complete the proof by induction, we need to use Lemma 7.2. Assume that the present lemma is true for $n=k$, and that $H$ is of class $\mathfrak{E}^{(k+1)}$ on $\mathfrak{U}_{p}$ uniformly $\left(\mathfrak{P} \mathfrak{U}_{p} \mathfrak{P}^{\prime} ; \sigma^{\prime}\right)$. Then $d_{u} H$ is of class $\mathfrak{S}^{(k)}$ on $\mathfrak{U}_{p}$ uniformly $\left(\mathfrak{P} \mathfrak{U}_{p} \mathfrak{P}^{\prime} \mathfrak{u}\right.$; $\left.\sigma^{\prime}\|d u\|\right)$, and hence of class $\mathfrak{E}^{(k)}$ on $\mathfrak{U}_{p}\left(u_{*}\right)_{a}$ uniformly $\left(\mathfrak{B} \mathfrak{U}_{p} \mathfrak{P}^{\prime}\left(u_{*}\right)_{a} ; \sigma^{\prime}\right)$, where $a$ is a positive number, by Lemma 7.2. Then by the present lemma for 
$n=k, d_{x} G$ is of class $\mathfrak{S}^{(k)}$ on $\mathfrak{X}_{0}\left(x_{*}\right)_{a}$ uniformly $\left(\mathfrak{X}_{0} \mathfrak{B}^{\prime}\left(x_{*}\right)_{a} ; \sigma^{\prime}\right)$. Application of Lemma 7.2 in the reverse direction now gives the desired conclusion.

The next lemma is concerned with reciprocal linear functions. We have a correspondence between functions $K_{1}$ on $\mathfrak{V}$ to $\mathfrak{V}$ which are linear on $\mathfrak{V}$ and such that $K_{1}(y \mid p)$ depends on $y$ only at $p$, and functions $K_{2}$ on $\mathfrak{B P}$ to $\mathfrak{B}$ which are linear on $\mathfrak{B}$ uniformly on $\mathfrak{B}$, by means of the equation

$$
K_{1}(y \mid p)=K_{2}(y(p), p) .
$$

It is evident that the function $K_{1}$ corresponding to such a function $K_{2}$ is actually linear on $\mathfrak{Y}$. In terms of this correspondence the lemma can be stated as follows.

LemMa 13.3. Let the function $K_{1}$ on $\mathfrak{Y}$ to $\mathfrak{V}$ be linear on $\mathfrak{Y}$, and such that $K_{1}(y \mid p)$ depends on $y$ only at $p$, and suppose that $K_{1}$ has a reciprocal $L_{1}$. Then $L_{1}$ necessarily has the same property as $K_{1}$. Moreover, the functions $K_{2}$ and $L_{2}$ on $\mathfrak{B P}$ to $\mathfrak{B}$ corresponding to $K_{1}$ and $L_{1}$ respectively, are reciprocal for every $p$ of $\mathfrak{B}$. Conversely, if $K_{2}$ and $L_{2}$ on $\mathfrak{B P}$ to $\mathfrak{B}$ are reciprocal for every $p$, and linear on $\mathfrak{B}$ uniformly on $\mathfrak{B}$, then the corresponding functions $K_{1}$ and $L_{1}$ are reciprocal. Finally, the moduli of $K_{1}$ and $K_{2}$ are equal, and the moduli of $L_{1}$ and $L_{2}$ are equal.

We first show that $K_{1}\left(y \mid p_{0}\right)=v_{*}$ if and only if $y\left(p_{0}\right)=v_{*}$. If $y\left(p_{0}\right)=v_{*}$, then $K_{1}\left(y \mid p_{0}\right)=K_{1}\left(y_{*} \mid p_{0}\right)=v_{*}$. If $K\left(y \mid p_{0}\right)=v_{*}$, define a function $y_{1}$ as follows: $y_{1}\left(p_{0}\right)=y\left(p_{0}\right), y_{1}(p)=v_{*}$ for $p \neq p_{0}$. Then $K_{1}\left(y_{1} \mid p_{0}\right)=K_{1}\left(y \mid p_{0}\right)=v_{*}$, and hence $K_{1}\left(y_{1}\right)=y_{*}$. Since $K_{1}$ has a reciprocal $L_{1}, y_{1}=y_{*}$, whence $y\left(p_{0}\right)=v_{*}$.

From this we can show that $L_{1}(y \mid p)$ depends on $y$ only at $p$. For, let $y_{1}(p)=y_{2}(p)$. Then

$$
\begin{aligned}
K_{1}\left(L_{1}\left(y_{1}-y_{2}\right) \mid p\right) & =y_{1}(p)-y_{2}(p)=v_{*}, \\
L_{1}\left(y_{1}-y_{2} \mid p\right) & =v_{*} .
\end{aligned}
$$

The reciprocal properties in the first part of the lemma and in the converse are practically obvious.

To show the equality of the moduli, we have, for $K_{1}$ and $K_{\mathbf{2}}$,

$$
\begin{aligned}
\left\|K_{1}(y)\right\| & =\bar{B}\left\|K_{1}(y \mid p)\right\|=\bar{B}\left\|K_{2}(y(p), p)\right\| \\
& \leqq M\left(K_{2}\right) \bar{B}\|y(p)\|=M\left(K_{2}\right)\|y\|, \\
\left\|K_{2}(v, p)\right\| & =\left\|K_{1}\left(y_{1} \mid p\right)\right\| \leqq\left\|K_{1}\left(y_{1}\right)\right\| \leqq M\left(K_{1}\right)\|v\|,
\end{aligned}
$$

where $\bar{B}$ denotes the upper bound as to $p$, and $y_{1}(p)=v$ for every $p$.

14. Solution of $B(x(p), y(p), p)=v_{*}$. If we set $\mathfrak{B}=(\mathfrak{u}, \mathfrak{B}), \mathfrak{B}=(\mathfrak{X}, \mathfrak{V})$, then $\mathbb{Z}$ and $\mathfrak{W}$ are related in the same way as $\mathfrak{U}$ and $\mathfrak{X}$. Also there corresponds 
to a region $\mathfrak{W}_{0}$ a system of regions $\mathfrak{Z}_{p}$ as defined in the opening paragraphs of this section. The following theorem is a corollary of Theorem IV of Paper I and will serve as a basis for the extended implicit function theorem of the next subsection. The principal point of the theorem is that there exist constants $a$ and $b$ independent of $p$.

ThEOREM VI. Consider a region $\mathfrak{W}_{0}$, and the corresponding system of regions $\mathfrak{B}_{p}$, a point $w_{0}=\left(x_{0}, y_{0}\right)$ of $\mathfrak{W}_{0}$, and a function $H$ on $\mathfrak{B}_{\mathfrak{B}_{p}}$ to $\mathfrak{B}$ with the following properties:

$\left(H_{1}\right) H\left(p, x_{0}(p), y_{0}(p)\right)=v_{*}$ for every $p$;

$\left(H_{2}\right) H$ is of class $\mathfrak{C}^{(n)}$ on $\AA_{p}$ uniformly on $\mathfrak{B} \mathcal{B}_{p}$;

$\left(H_{3}\right) d_{v} H\left(p, x_{0}(p), y_{0}(p) ; d v\right) \equiv K_{0}(d v, p)$ has a reciprocal $L_{0}$, and $L_{0}$ is linear on $\mathfrak{B}$ uniformly on $\mathfrak{B}$.

Under these hypotheses there exist positive constants $a$ and $b$ and $a$ function $\mathfrak{B}$ on $\mathfrak{B}\left(x_{0}(p)\right)_{b}$ to $\mathfrak{B}$ with the following properties:

$\left(C_{1}\right)$ the region $\left(\left(x_{0}(p)\right)_{b},\left(y_{0}(p)\right)_{a}\right.$ is contained in $3_{p}$ for every $p$;

$\left(C_{2}\right)$ for every $p$ and every $u$ in $\left(x_{0}(p)\right)_{b}, V(p, u)$ is the unique solution of the equation

$$
H(p, u, v)=v_{*},
$$

having $v$ in $\left(y_{0}(p)\right)_{a}$;

$\left(C_{3}\right)$ the differential $d_{v} H(p, u, V(p, u) ; d v)$ has a reciprocal $L$ on $\mathfrak{B P}\left(x_{0}(p)\right)_{b}$ to $\mathfrak{B}$, which is linear on $\mathfrak{B}$ uniformly on $\mathfrak{B}\left(x_{0}(p)\right)_{b}$;

$\left(C_{4}\right)$ the function $V$ is of class $\mathfrak{S}^{(n)}$ on $\left(x_{0}(p)\right)_{b}$ uniformly on $\mathfrak{B}\left(x_{0}(p)\right)_{b}$.

We reduce this theorem to Theorem IV of Paper I by considering the function $G$ on $\mathfrak{W}_{0}$ defined by

$$
G(x, y \mid p)-H(p, x(p), y(p)) .
$$

If the functional values of $G$ are to be in the space $\mathfrak{V},\|H(p, x(p), y(p))\|$ must be bounded on $\mathfrak{B}$. This will be so if the point $(x, y)$ lies in a neighborhood $\left(\left(x_{0}, y_{0}\right)\right)_{c}$ which in turn lies in the region $\mathfrak{W}_{0}$. To prove this we apply the hypotheses $\left(H_{1}\right)$ and $\left(H_{2}\right)$ and Taylor's theorem.*

After we replace the region $\mathfrak{B}_{0}$ by the neighborhood $\left(w_{0}\right)_{c}$, we can readily show by Lemmas 13.2 and 13.3 that the point $w_{0}$ and the function $G$ on $\left(w_{0}\right)_{c}$ to $\mathfrak{V}$ satisfy all the hypotheses of Theorem IV of Paper I.

The next step is to show that the function $Y$ obtained from Theorem IVI is such that $Y(x \mid p)$ depends on $x$ only at $p$. Suppose that for a certain $p_{0}$ we have

\footnotetext{
* See Paper II.
}

$$
x_{1}\left(p_{0}\right)=x_{2}\left(p_{0}\right), \quad Y\left(x_{1} \mid p_{0}\right) \neq Y\left(x_{2} \mid p_{0}\right) .
$$


Then a second solution $\bar{Y}\left(x_{1}\right)$, in $\left(y_{0}\right)_{a}$, but not equal to $Y\left(x_{1}\right)$, is given by

$$
\bar{Y}\left(x_{1} \mid p_{0}\right)=Y\left(x_{2} \mid p_{0}\right), \quad \bar{Y}\left(x_{1} \mid p\right)=Y\left(x_{1} \mid p\right) \quad\left(p \neq p_{0}\right) .
$$

And this contradicts the uniqueness of the solution.

If we set $V(p, u)=V(x \mid p)$, where $x(p)=u$, then the function $V$ and the constants $a$ and $b$ obviously satisfy the conditions $\left(C_{1}\right)$ and $\left(C_{2}\right)$. The conclusion $\left(C_{3}\right)$ follows from Lemma 13.3, and $\left(C_{4}\right)$ from Lemma 13.1 and the remark appended thereto.

15. An extended implicit function theorem. The theorem we shall obtain is a generalization of those given by Bolza and others, but our method of proof is entirely different. The theorem is "extended" in the sense that there is a set of initial solutions instead of only a single initial solution, and that there is a uniform neighborhood of the initial set in which there is a unique solution.

If $\mathfrak{u}^{(0)}$ is a set of points in the linear metric space $\mathfrak{u}$, we shall denote such a uniform neighborhood by $\left(\mathfrak{u}^{(0)}\right)_{a}$, as in $\S 5$.

We shall be considering a set $\mathfrak{Z}^{(0)}$ in the composite space $\mathbb{Z}=(\mathfrak{U}, \mathfrak{B})$, having the property that if $\left(u_{1}, v_{1}\right),\left(u_{2}, v_{2}\right)$ are distinct points of $\mathfrak{Z}^{(0)}$, then $u_{1} \neq u_{2}$. Let $\mathfrak{u}^{(0)}$ be the "projection" of $\mathfrak{Z}^{(0)}$ on the space $\mathfrak{u}$. Then such a set $\mathfrak{Z}^{(0)}$ defines a single-valued function $V^{(0)}$ on $\mathfrak{U}^{(0)}$ to $\mathfrak{B}$.

ThEOREM VII. Let $\mathfrak{Z}^{(0)}$ be a set of points defining a single-valued function $V^{(0)}$ on $\mathfrak{u}^{(0)}$, as just described, and let $G$ be a function on a region $\mathfrak{Z}_{0}$ to $\mathfrak{B}$, with the following properties:

$\left(H_{1}\right) \mathfrak{Z}^{(0)}$ is in $3_{0}$;

$\left(H_{2}\right) G(z)=G(u, v)=v_{*}$ on $3^{(0)}$;

$\left(H_{3}\right) G$ is of class $\mathbb{E}^{(n)}$ on $Z_{0}$ uniformly on $Z_{0}$;

$\left(H_{4}\right) d_{v} G(z ; d v)$ has a reciprocal $L(z ; d v)$ for every $z$ in $3^{(0)}$;

$\left(H_{5}\right)$ the set $\mathfrak{B}^{(0)}$ is bounded;

$\left(H_{6}\right)$ there is a neighborhood $\left(\mathfrak{B}^{(0)}\right)_{c}$ in $3_{0}$;

$\left(H_{7}\right)$ the function $V^{(0)}$ is continuous on $\mathfrak{u}^{(0)}$ uniformly on $\mathfrak{u}^{(0)}$;

$\left(H_{8}\right) L(z ; d v)$ is linear on $\mathfrak{B}$ uniformly on $\mathfrak{B}^{(0)}$.

Then there exist positive constants $a$ and $b$ and a function $V$ on $\left(\mathfrak{U}^{(0)}\right)_{b}$ to $\mathfrak{B}$ with the following properties:

$\left(C_{1}\right)\left(B^{(0)}\right)_{a}$ is in $\mathbb{B}_{0}$;

$\left(C_{2}\right)$ for every $u$ in $\left(\mathfrak{u}^{(0)}\right)_{b}, V(u)$ is the unique solution of the equation $G(u \quad v)=v_{*}$ for which $(u, v)$ is in $\left(B^{(0)}\right)_{a}$;

$\left(C_{3}\right)$ the function $V$ is of class $\mathfrak{C}^{(n)}$ on the region $\left(\mathfrak{u}^{(0)}\right)_{b}$.

In case the set $\mathfrak{Z}^{(0)}$ is self-compact, the hypotheses $\left(H_{6}\right)$ to $\left(H_{8}\right)$ may be omitted. 
We reduce this theorem to Theorem VI by setting $\mathfrak{B}=\mathfrak{Z}^{(0)}, p=(u, v)$, where $(u, v)$ is a point of $Z^{(0)}$. Corresponding to this range $\mathfrak{B}$ and the spaces $\mathfrak{U}$ and $\mathfrak{B}$, we define the spaces $\mathfrak{X}$ and $\mathfrak{Y}$ as at the beginning of this section. The initial solution $\left(x_{0}, y_{0}\right)$ is defined by

$$
\begin{aligned}
& x_{0}(p)=u \text {-component of } p \equiv u_{p}, \\
& y_{0}(p)=v \text {-component of } p \equiv v_{p} .
\end{aligned}
$$

The region $\mathfrak{W}_{0}$ consists of all points $w=(x, y)$ for which there is a uniform neighborhood $(w(p))_{d}$ in $Z_{0}$ for every $p$. Then by Theorem VI there exist positive constants $a$ and $b$, and a function $V_{1}$ on $\mathfrak{B}\left(u_{p}\right)_{b}$ to $\mathfrak{B}$ such that

(1) $\left(\left(u_{p}\right)_{b},\left(v_{p}\right)_{a}\right)$ is contained in $Z_{0}$ for every $p$;

(2) for every $p$ and every $u$ in $\left(u_{p}\right)_{b}, V_{1}(p, u)$ is the unique solution of the equation $G(u, v)=v_{*}$ having $v$ in $\left(v_{p}\right)_{a}$;

(3) the function $V_{1}$ is of class $\mathfrak{S}^{(n)}$ on $\left(u_{p}\right)_{b}$ uniformly on $\mathfrak{B}\left(u_{p}\right)_{b}$.

We have now to show that when the constants $a$ and $b$ are replaced by constants $a^{\prime}$ and $b^{\prime}$ sufficiently small, the function $V_{1}$ is independent of $p$ when $u$ is in $\left(\mathfrak{u}^{(0)}\right)_{b^{\prime}}$ and yields the unique solution having $(u, v)$ in $\left(\mathbb{B}^{(0)}\right)_{a^{\prime}}$. The hypothesis $\left(H_{7}\right)$, put in explicit form, reads as follows: for every positive number $\epsilon$ there is a positive $\delta$ such that, when the distance of two points $u_{1}$ and $u_{2}$ of $\mathfrak{u}^{(0)}$ is less than $\delta$, we have $\left\|V^{(0)}\left(u_{1}\right)-V^{(0)}\left(u_{2}\right)\right\|<\epsilon$. Let $\delta_{1}$ correspond to $\epsilon=a / 2$, and let $a^{\prime}$ be the smallest of the numbers $a / 2, \delta_{1} / 2$, and $b$. Then since the function $V_{1}$ is continuous on $\left(u_{p}\right)_{b}$ uniformly on $\mathfrak{B}\left(u_{p}\right)_{b}$, the constant $a$ may be replaced by $a^{\prime}$ in the statements above if $b$ is replaced by a constant $b^{\prime}$ sufficiently small. Furthermore we may take $b^{\prime} \leqq a^{\prime}$. Now suppose $\left(u, v_{1}\right)$ and $\left(u, v_{2}\right)$ are two solutions of the equation $G(u, v)=v_{*}$, both in $\left(\mathfrak{S}^{(0)}\right)_{a^{\prime}}$. That is, $\left(u, v_{1}\right)$ is in a neighborhood $\left(\left(u_{01}, v_{01}\right)\right)_{a^{\prime}}$ and $\left(u, v_{2}\right)$ is in a neighborhood $\left(\left(u_{02}, v_{02}\right)\right)_{a^{\prime}}$, where $\left(u_{01}, v_{01}\right)$ and $\left(u_{02}, v_{02}\right)$ are points of $\mathfrak{Z}^{(0)}$. Then we have, in succession,

$$
\begin{aligned}
\left\|u_{01}-u_{02}\right\| & <2 a^{\prime} \leqq \delta_{1}, \\
\left\|v_{01}-v_{02}\right\| & <\epsilon=a / 2, \\
\left\|v_{01}-v_{2}\right\| & <a / 2+a^{\prime} \leqq a,
\end{aligned}
$$

so that $v_{1}$ and $v_{2}$ are both in $\left(v_{01}\right)_{a}$. Therefore $v_{1}=v_{2}=V_{1}(p, u)$, where $p=$ $\left(u_{01}, v_{01}\right)$. Hence on the region $\left(\mathfrak{u}^{(0)}\right)_{b}$, we may set $V_{1}(u, p)=V(u)$.

Finally, from the differentiability properties of the function $V_{1}$ we find that the function $V$ is of class $\mathfrak{S}^{(n)}$ on the region $\left(\mathfrak{U}^{(0)}\right)_{b^{\prime}}$.

In case the set $\mathbf{3}^{(0)}$ is self-compact, the hypotheses $\left(H_{5}\right)$ and $\left(H_{6}\right)$ follow from Lemmas 5.1 and 5.2. The hypothesis $\left(H_{7}\right)$ is obtained immediately by an indirect proof. To obtain $\left(H_{8}\right)$ from Lemma 5.5, we need to show first 
that the reciprocal $L$ of $d_{v} G$ has the same continuity as $d_{v} G$ on the set $Z^{(0)}$. The proof for this is formally the same as that in Lemma 16.2 of Paper I.

\section{Extension of the domain of Definition of a FUNCTion, WITH PRESERVATION OF A LIPSCHITZ CONDITION}

16. In an existence theorem for the solution of a differential equation, it is desirable to show that the solution extends to the boundary of the domain of definition of the functions involved in the differential equation. As Professor Jackson has remarked,* this can easily be done by extending the domain of definition of the functions to include the whole space of the dependent variables. However, this method yields more restricted results than can be obtained by other methods. $\dagger$

The extension of the domain of definition can readily be carried out in our abstract spaces in case the original domain is a neighborhood. Extensions from more general domains with preservation of continuity have been given, $\ddagger$ but it does not seem possible to prove that those extensions preserve a Lipschitz condition.

Consider a linear metric space $\mathfrak{X}$ and a complete linear metric space $\mathfrak{Z}$, and a function $F$ on a region $\mathfrak{X}_{0}$ to $\mathfrak{Y}$. We say that $F$ satisfies a Lipschitz condition with constant $k$ on $\mathfrak{X}_{0}$ in case for every $x_{1}$ and $x_{2}$ in $\mathfrak{X}_{0}$ we have

$$
\left\|F\left(x_{1}\right)-F\left(x_{2}\right)\right\| \leqq k\left\|x_{1}-x_{2}\right\| .
$$

THEOREM VIII. If a function $F$ on a neighborhood $\left(x_{0}\right)_{a}$ to $\supseteqq$ satisfies a Lipschitz condition with constant $k$ on $\left(x_{0}\right)_{a}$, then there exists a function $G$ on $X$ to $\mathfrak{V}$, equal to $F$ on $\left(x_{0}\right)_{a}$, and satisfying a Lipschitz condition with constant $2 k$ on $\mathfrak{X}$.

If the function $F$ is on $\left(x_{0}\right)_{a} \mathfrak{P}$ to $\mathfrak{V}$, we evidently have the theorem for each $p$ of $\mathfrak{P}$, whether $k$ depends on $p$ or not. For example, $\mathfrak{P}$ might be a region $\mathfrak{B}_{0}$ of a metric space $\mathfrak{B}$. It is also evident that the function $G$ of the theorem is not unique, and that the constant $2 k$ for $G$ is larger than necessary (at least in most cases).

If a function $F$ on a set $\mathfrak{X}^{(0)}$ to $\mathfrak{Y}$ is continuous on $\mathfrak{X}^{(0)}$ uniformly on $\mathfrak{X}^{(0)}$, then a function $H$ on $\mathfrak{X}^{(0)}$ plus its boundary is uniquely defined by the

\footnotetext{
- See Annals of Mathematics, (2), vol. 23 (1922), p. 75.

$\dagger$ Cf. Bliss, The solutions of differential equations of the first order as functions of their initial values, Annals of Mathematics, (2), vol. 6 (1905), p. 49. Sec. 125.

† Cf. Tietze, Crelle's Journal, vol. 145 (1915), p. 9; de la Vallée Poussin, Intégrales de Lebesgue,
} 
properties that $H$ equals $F$ on $\mathfrak{X}^{(0)}$, and $H$ is continuous on $\mathfrak{X}^{(0)}$ plus its boundary. This has been shown by Fréchet* by the usual methods for the case when $\mathfrak{Y}$ is the real number system, and the proof is exactly the same for our general case.

Thus we have a uniquely determined function $H$ on $\left[x_{0}\right]_{a}$ to $\mathfrak{V}$ (where $\left[x_{0}\right]_{a}$ denotes the neighborhood $\left(x_{0}\right)_{a}$ plus its boundary). It is readily verified that the same constant $k$ is valid for $H$ on $\left[x_{0}\right]_{a}$ as for $F$ on $\left(x_{0}\right)_{a}$. We now define the function $G$, for points $x$ not in the set $\left[x_{0}\right]_{a}$, by the formula

$$
G(x)=H\left(x_{0}+\frac{\left(x-x_{0}\right) a}{\left\|x-x_{0}\right\|}\right) .
$$

To show that $G$ satisfies a Lipschitz condition, we simplify the formulas by the transformation $x=x_{0}+\bar{x} a$. This makes the set $\left[x_{0}\right]_{a}$ correspond to the set $\left[x_{*}\right]_{1}$, and the formula (16.1) becomes

$$
G(x)=H\left(\frac{x}{\|x\|}\right)
$$

for points $x$ not in $\left[x_{*}\right]_{1}$. Take first the case $\left\|x_{1}\right\|>1,\left\|x_{2}\right\|>1$. We have

$$
\begin{aligned}
& \left\|G\left(x_{1}\right)-G\left(x_{2}\right)\right\|=\left\|H\left(\frac{x_{1}}{\left\|x_{1}\right\|}\right)-H\left(\frac{x_{2}}{\left\|x_{2}\right\|}\right)\right\| \\
& \leqq k\left\{\left\|\frac{x_{1}}{\left\|x_{1}\right\|}-\frac{x_{2}}{\left\|x_{2}\right\|}\right\|\right\} \leqq k\left\{\left\|\frac{x_{1}}{\left\|x_{1}\right\|}-\frac{x_{2}}{\left\|x_{1}\right\|}\right\|+\left\|\frac{x_{2}}{\left\|x_{1}\right\|}-\frac{x_{2}}{\left\|x_{2}\right\|}\right\|\right\} \\
& \quad=\frac{k}{\left\|x_{1}\right\|}\left\{\left\|x_{1}-x_{2}\right\|+\|\| x_{2}\|-\| x_{1} \| \mid\right\}<2 k\left\|x_{1}-x_{2}\right\| .
\end{aligned}
$$

The manipulation is similar in case one of the points is in $\left[x_{*}\right]_{1}$, while the other is outside.

\section{IMBEDDING THEOREMS FOR DIFFERENTIAL EQUATIONS}

The equations to be considered are called differential equations for convenience; they include both ordinary differential equations and Volterra integral equations as special cases. The form of the equations is the same as in Paper I, but a different kind of Lipschitz condition is assumed. Consequently a new convergence proof is required. $\dagger$ However, Theorems IV and V

\footnotetext{
* Bulletin des Sciences Mathématiques, (2), vol. 48 (1924), p. 171.

$\dagger$ For an illuminating discussion of the close connection between the two types of theorems, see Evans, Cambridge Colloquium, pp. 52-54.
} 
of Paper I can be and are applied to the differential equations considered in this section, the new convergence proof being essential in showing the existence of a reciprocal for $d_{y} G$.

17. Notations. We consider a bounded and measurable set $\Re^{(0)}$ of points of the real axis $\Re$, and a linear metric space $\mathfrak{B}$. Let $\mathfrak{Z}$ be a class of functions $y$ on $\Re^{(0)}$ to $\mathfrak{B}$, constituting a complete linear metric space of type $A_{0}$, with $\Re=\Re^{(0)}$, and $\sigma(p)=1$ for every $p$. Let $r_{0}$ be a fixed point of $\Re^{(0)}$ For a point $y$ of the space $\mathfrak{Z}$ we shall set

$$
\int^{r}\|y(r)\|=\text { the greatest lower bound of }\left|\int_{r_{0}}^{r} R(r) d r\right|,
$$

for all functions $R$ on $\Re^{(0)}$ to $\Re$ which are continuous on $\Re^{(0)}$ and such that $R(r) \geqq\|y(r)\|$ for every $r$. The integral on the right is understood to be taken over the points of $\Re^{(0)}$ contained in the interval $\left(r_{0} r\right)$. We might suppose the function $R$ to be merely integrable in the sense of Lebesgue, but that would unnecessarily strengthen the hypotheses of the theorems to follow. In many special cases the function $\|y(r)\|$ is itself measurable, but that is not necessarily so in general. In case the set $\Re^{(0)}$ has measure zero, all the results are trivial, but still valid.

18. General imbedding theorems. The first theorem corresponds to Theorem I of Paper I.

THEOREM IX. Let the point $y_{0}$ of $\mathfrak{Y}$ and the region $\mathfrak{X}_{0}$ of the metric space $\mathfrak{X}$, and the function $F$ on $\mathfrak{X}_{0}\left(y_{0}\right)_{a}$ to $\mathfrak{Y}$ be such that

$\left(H_{1}\right)$ for every $x$ in $\mathfrak{X}_{0}$ there is a constant $k_{x}>0$ such that, whenever $y_{1}$ and $y_{2}$ are in $\left(y_{0}\right)_{a}$ and $r$ is in $\Re^{(0)}$ we have

$$
\left\|F\left(x, y_{1} \mid r\right)-F\left(x, y_{2} \mid r\right)\right\| \leqq k_{x} \int^{r}\left\|y_{1}(r)-y_{2}(r)\right\| ;
$$

$\left(H_{2}\right)$ for every $x$ in $\mathfrak{X}_{0}$ we have

$$
\left\|F\left(x, y_{0}\right)-y_{0}\right\|<a e^{-k_{x} d}
$$

where $d$ is the diameter of the set $\Re^{(0)}$.

Then there exists a unique function $Y$ on $\mathfrak{X}_{0}$ to $\left(y_{0}\right)_{a}$ such that

for every $x$ in $\mathfrak{X}_{0}$.

$$
Y(x)=F(x, Y(x))
$$

Following the method of Part V, we could in many special cases extend the range of definition of the function $F$ to include the whole $\mathfrak{V}$ space. The hypothesis $\left(H_{2}\right)$, which serves to make sure that all the approximations and 
their limit are in $\left(y_{0}\right)_{a}$, could then be omitted. However, the conclusion would also have to be modified.

It is noticeable that the nature of the range $\mathfrak{X}_{0}$ is not essential in the theorem, and the theorem is in fact unchanged in content if $\mathfrak{X}_{0}$ is replaced by a general range $\mathfrak{B}$ or else omitted altogether. The case is otherwise in the later theorems where we consider continuity and differentiability with respect to $x$. In applications, $x$ may represent initial values or parameters or a combination of the two. In the proof of the present theorem we shall omit to write the argument $x$.

As usual we define a sequence of approximations by the equations

$$
y_{1}=F\left(y_{0}\right), \quad y_{n+1}=F\left(y_{n}\right) .
$$

To show that all the approximations are surely defined, we have

$$
\left\|y_{1}-y_{0}\right\|=c<a e^{-k d}<a,\left\|y_{n+1}(r)-y_{n}(r)\right\| \leqq k \int^{r}\left\|y_{n}(r)-y_{n-1}(r)\right\|,
$$

from which, by induction,

$$
\left\|y_{n+1}(r)-y_{n}(r)\right\| \leqq \frac{c\left(k\left|r-r_{0}\right|\right)^{n}}{n !} .
$$

Therefore we have, for every $n$,

$$
\left\|y_{n}-y_{0}\right\| \leqq c e^{k d}<a .
$$

The inequality (18.1) shows at the same time the convergence of the sequence $\left\{y_{n}\right\}$ to an element $y$ of $\mathfrak{y}$. To show that this limit $y$ is a solution of the equation, we have

$$
\begin{aligned}
& \|y(r)-F(y \mid r)\| \leqq\left\|y(r)-y_{n+1}(r)\right\|+\left\|F\left(y_{n} \mid r\right)-F(y \mid r)\right\| \\
& \quad \leqq\left\|y-y_{n+1}\right\|+k \int^{r}\left\|y_{n}(r)-y(r)\right\| \leqq\left\|y-y_{n+1}\right\|+k d\left\|y_{n}-y\right\| .
\end{aligned}
$$

To show the uniqueness of the solution we have, if $y_{1}$ and $y_{2}$ are solutions,

$$
\left\|y_{1}(r)-y_{2}(r)\right\|=\left\|F\left(y_{1} \mid r\right)-F\left(y_{2} \mid r\right)\right\| \leqq k \int^{r}\left\|y_{1}(r)-y_{2}(r)\right\|,
$$

and by induction,

$$
\left\|y_{1}(r)-y_{2}(r)\right\| \leqq \frac{\left(k\left|r-r_{0}\right|\right)^{n}}{n !}\left\|y_{1}-y_{2}\right\| .
$$

For $n$ sufficiently large, this contradicts $y_{1} \neq y_{2}$.

The next theorem corresponds to Lemma 16.1 of Paper I. 
TheOREM X. Suppose the function $G$ on $\mathfrak{Y}$ to $\mathfrak{V}$ is distributive on $\mathfrak{V}$ and is such that there exists a constant $M$ for which

$$
\|G(y \mid r)\| \leqq M \int^{r}\|y(r)\|
$$

for every $r$ and $y$. Then the function $K$ on $\mathfrak{Y}$ to $\mathfrak{Z}$ defined by

$$
K(y)=y-G(y)
$$

is linear on $\mathfrak{V}$ and has a reciprocal $L$. Moreover the modulus of $L$ is not greater than $e^{M d}$, where $d$ is the diameter of $\Re^{(0)}$.

The linearity of $K$ is evident. If we apply Theorem IX to the equation

$$
y=G(y)+y^{\prime},
$$

we find that there exists a unique function $L$ on $\mathfrak{V}$ to $\mathfrak{y}$ such that $K\left(L\left(y^{\prime}\right)\right)=y^{\prime}$. From the uniqueness we have $L(K(y))=L\left(y^{\prime}\right)=y$.

It remains to show that $L$ is linear. The distributive property of $L$ follows from the form of equation (18.2) and the fact that $L$ is its unique solution. To show that $L$ is modular, we consider the series

$$
\sum_{n=0}^{\infty} G_{n}(y), \text { where } G_{0}(y)=y, G_{n+1}(y)=G\left(G_{n}(y)\right),
$$

of which $L$ is the sum. By induction we readily find that

$$
\left\|G_{n}(y \mid r)\right\| \leqq \frac{\left(M\left|r-r_{0}\right|\right)^{n}}{n !}\|y\|
$$

and hence $\|L(y)\| \leqq e^{M d}\|y\|$, where $d$ is the diameter of $\Re^{(0)}$.

The next theorem has similarities to Theorems $\mathrm{II}^{\mathrm{I}}, \mathrm{IV}^{\mathrm{I}}$, and $\mathrm{V}^{\mathrm{I}}$ (of Paper I), and is proved on the basis of Theorems IVI, VI, and Theorem X.

ThEOREM XI. Let $\mathfrak{X}$ be a linear metric space, and let $\mathfrak{W}_{0}$ be a convex region of the space $\mathfrak{W}=(\mathfrak{X}, \mathfrak{V})$. Suppose the point $w_{0}=\left(x_{0}, y_{0}\right)$ of $\mathfrak{W}_{0}$ and the function $F$ on $\mathfrak{W}_{0}$ to $\mathfrak{V}$ are such that

$\left(H_{1}\right)\left(x_{0}, y_{0}\right)$ is a solution of the equation

$$
y=F(x, y)
$$

$\left(H_{2}\right) F$ is of class $\mathfrak{C}^{(n)}$ on $\mathfrak{W}_{0}$;

$\left(H_{3}\right)$ the differential $d_{y} F$ satisfies the condition that for every $x$ there exists a constant $M_{x}$ such that, whenever $(x, y)$ is in $\mathfrak{B}_{0}$, we have

$$
\left\|d_{y} F(x, y ; d y \mid r)\right\| \leqq M_{x} \int^{r}\|d y(r)\|
$$


for every r of $\Re^{(0)}$ and every dy in $\mathfrak{Y}$.

Then there exist a unique region $\mathfrak{X}_{0}$ containing the point $x_{0}$ and a unique function $Y$ on $\mathfrak{X}_{0}$ to $\mathfrak{V}$ with the following properties:

$\left(C_{1}\right) \mathfrak{X}_{0}$ is connected;

$\left(C_{2}\right)$ for every $x$ in $\mathfrak{X}_{0},(x, Y(x))$ is in $\mathfrak{W}_{0}$, and satisfies the equation (18.3);

$\left(C_{3}\right) Y$ is of class $\mathcal{E}^{(n)}$ on $\mathfrak{X}_{0}$;

$\left(C_{4}\right)$ if $x^{\prime}$ is a boundary point of the region $\mathfrak{X}_{0}$, and if $Y(x)$ has a limit $y^{\prime}$ for $x=x^{\prime}$, then $\left(x^{\prime}, y^{\prime}\right)$ is a boundary point of the region $\mathfrak{W}_{0}$.

To apply Theorems IVI and VI, we set

$$
y-F(x, y)=G(x, y) \text {. }
$$

Then by Theorem $\mathrm{X}, d_{y} G$ has a reciprocal for every point $(x, y)$ in $\mathfrak{B}_{0}$. Hence every point of $\mathfrak{W}_{0}$ is an ordinary point for the function $G$. Also there is a unique sheet $\mathfrak{B}^{(0)}$ of solutions, with the properties described in Theorem VI.

We next show that there cannot be two solutions $y_{1}$ and $y_{2}$ for a given $x$, so that the sheet $\mathfrak{W}^{(0)}$ is single-valued. For, suppose that there were two solutions. Then by Theorem I ( $\$ 4)$, Taylor's theorem, ${ }^{*}$ and the hypothesis $\left(H_{3}\right)$ we have

$$
\begin{aligned}
\left\|y_{1}(r)-y_{2}(r)\right\| & =\left\|F\left(x, y_{1} \mid r\right)-F\left(x, y_{2} \mid r\right)\right\| \\
& =\left\|\int_{0}^{1} d_{y} F\left(x, y_{2}+\left(y_{1}-y_{2}\right) s ; y_{1}-y_{2} \mid r\right) d s\right\| \\
& \leqq M_{x} \int^{r}\left\|y_{1}(r)-y_{2}(r)\right\| .
\end{aligned}
$$

From this we obtain by induction

$$
\left\|y_{1}(r)-y_{2}(r)\right\| \leqq\left\|y_{1}-y_{2}\right\| \frac{\left(M_{x}\left|r-r_{0}\right|\right)^{n}}{n !},
$$

which is a contradiction for $n$ sufficiently large.

By the definition of a sheet, the projection of $\mathfrak{W}^{(0)}$ on the space $X$ is a connected region $\mathfrak{X}_{0}$. Then the sheet $\mathfrak{W}^{(0)}$ defines a single-valued function $Y$ on $\mathfrak{X}_{0}$ to $\mathfrak{V}$ satisfying the conclusions $\left(C_{1}\right)$ to $\left(C_{3}\right)$.

A point $\left(x^{\prime}, y^{\prime}\right)$ described in $\left(C_{4}\right)$ is certainly a boundary point of the sheet $\mathfrak{W}^{(0)}$, and hence of the region $\mathfrak{W}_{0}$, since all the points of $\mathfrak{W}_{0}$ are ordinary.

Suppose there were two regions $\mathfrak{X}_{01}$ and $\mathfrak{X}_{02}$ and corresponding functions $Y_{1}$ and $Y_{2}$, satisfying the conclusions of the theorem. Then $Y_{1}=Y_{2}$ on the

* See Paper II. 
region common to $\mathfrak{X}_{01}$ and $\mathfrak{X}_{02}$. Let $x_{1}$ be a point of $\mathfrak{X}_{01}$, connected to $x_{0}$ by a function $X$ continuous on the interval (01) to $\mathfrak{X}_{01}$. Let $s^{\prime}$ be the upper bound of the points $s$ on (01) such that $X(s)$ is in $\mathfrak{X}_{02}$. If $X\left(s^{\prime}\right)$ is not in $\mathfrak{X}_{02}$ (which is certainly true if $\left.s^{\prime} \neq 1\right)$, then $X\left(s^{\prime}\right)$ is a boundary point of $\mathfrak{X}_{02}$, and

$$
\lim _{x=X\left(s^{\prime}\right)} Y_{2}(x)=Y_{1}\left(X\left(s^{\prime}\right)\right) .
$$

Hence by $\left(C_{4}\right),\left(X\left(s^{\prime}\right), Y_{1}\left(X\left(s^{\prime}\right)\right)\right)$ is a boundary point of the region $\mathfrak{B}_{0}$, which contradicts $\left(C_{2}\right)$. Hence all the points $x_{1}$ of $\mathfrak{X}_{01}$ are contained in $\mathfrak{X}_{02}$. Similarly $\mathfrak{X}_{01}$ contains $\mathfrak{X}_{02}$.

The following modification of the last theorem is interesting, and follows from Theorem X and Theorem IV'.

THEOREM $\mathrm{XI}^{\prime}$. In case the hypothesis $\left(\mathrm{H}_{3}\right)$ is satisfied only at the initial solution $\left(x_{0}, y_{0}\right)$, then there exist positive constants $a$ and $b$ and $a$ unique function $Y$ on $\left(x_{0}\right)_{b}$ to $\left(y_{0}\right)_{a}$ with the properties $\left(C_{2}\right)$ and $\left(C_{3}\right)$.

In stating still another theorem following from Theorems $\mathrm{X}$ and IVI, we consider a space $B$ of functions $z$ on $\Re^{(0)}$ to a linear metric space $\mathfrak{U}$. The space $\mathbb{Z}$ is to have all the properties assumed for the space $\mathfrak{V}$, except that it need not be complete.

THEOREM XII. Consider a function $F$ on a region $(\mathfrak{X}, \mathfrak{Y}, \mathfrak{Z})_{0}$ of the composite space $(\mathfrak{X}, \mathfrak{Y}, \mathfrak{Z})$, to $\mathfrak{Y}$, a function $H$ on a region $(\mathfrak{X}, \mathfrak{Y})_{1}$ of the space $(\mathfrak{X}, \mathfrak{Y})$, to 3 , and a point $\left(x_{0}, y_{0}, z_{0}\right)$, with the following properties:

$\left(H_{1}\right)\left(x_{0}, y_{0}\right)$ is in $(\mathfrak{X}, \mathfrak{Y})_{1}$, and $\left(x_{0}, y_{0}, z_{0}\right)$ is in $(\mathfrak{X}, \mathfrak{Y}, \mathfrak{Z})_{0}$;

$\left(H_{2}\right) H\left(x_{0}, y_{0}\right)=z_{0}, F\left(x_{0}, y_{0}, z_{0}\right)=y_{*}$;

$\left(H_{3}\right) F$ and $H$ are of class $\mathfrak{E}^{(n)}$ on their respective domains of definition [uniformly on those domains];

$\left(H_{4}\right)$ the differential $d_{y} F\left(x_{0}, y_{0}, z_{0} ; d y\right)$ has a reciprocal $L_{0}$;

$\left(H_{5}\right)$ there exists a positive constant $k$ such that, for every $r$ in $\Re^{(0)}$, every $d y$ in $\mathfrak{Y}$ and every $d z$ in 3 , we have

$$
\begin{aligned}
\left\|d_{y} H\left(x_{0}, y_{0} ; d y \mid r\right)\right\| & \leqq k \int^{r}\|d y(r)\|, \\
\left\|d_{z} F\left(x_{0}, y_{0}, z_{0} ; d z \mid r\right)\right\| & \leqq k\|d z(r)\|, \\
\left\|L_{0}(d y \mid r)\right\| & \leqq k\|d y(r)\| .
\end{aligned}
$$

Then there exist positive constants $a$ and $b$, and a unique function $Y$ on $\left(x_{0}\right)_{b}$ to $\left(y_{0}\right)_{a}$, with the following properties:

$\left(C_{1}\right)$ the function

$$
G(x, y)=F(x, y, H(x, y))
$$

is defined for $x$ in $\left(x_{0}\right)_{b}$, and $y$ in $\left(y_{0}\right)_{a}$; 
$\left(C_{2}\right)$ for every $x$ in $\left(x_{0}\right)_{b}$ the point $(x, Y(x))$ satisfies the equation

$$
G(x, y)=F(x, y, H(x, y))=y_{*} ;
$$

$\left(C_{3}\right)$ the function $Y$ is of class $\mathbb{S}^{(n)}$ on $\left(x_{0}\right)_{b}$ [uniformly on $\left(x_{0}\right)_{b}$ ].

Since a region consists wholly of interior points, and the function $B$ is continuous, the conclusion $\left(C_{1}\right)$ is evidently true whenever the constants $a$ and $b$ are sufficiently restricted. The function $G$ is of class $\mathbb{E}^{(n)}$ on $\left(x_{0}\right)_{b}\left(y_{0}\right)_{a}$ [uniformly on $\left(x_{0}\right)_{b}\left(y_{0}\right)_{a}$ ] by Lemma 15.3 of Paper I. To show that $d_{y} G$ has a reciprocal at $\left(x_{0}, y_{0}\right)$, set

$$
\begin{aligned}
& K_{1}(d y)=d_{y} F\left(x_{0}, y_{0}, z_{0} ; d y\right), \\
& K_{2}(d y)=d_{z} F\left(x_{0}, y_{0}, z_{0} ; d_{y} H\left(x_{0}, y_{\theta} ; d y\right)\right), \\
& K_{3}(d y)=d_{y} G\left(x_{0}, y_{0} ; d y\right), \\
& K_{4}(d y)=-L_{0}\left(K_{2}(d y)\right) .
\end{aligned}
$$

Then $K_{3}=K_{1}+K_{2}$, and $L_{0}$ is the reciprocal of $K_{1}$. The linear function $K_{4}$ satisfies the conditions of Theorem $\mathrm{X}$, since

$$
\left\|K_{4}(d y \mid r)\right\| \leqq k^{3} \int^{r}\|d y(r)\| .
$$

Let $K_{5}$ be the reciprocal of $d y-K_{4}(d y)$. Then $K_{5}\left(L_{0}(d y)\right)$ is the reciprocal of $K_{3}(d y)=d_{y} G\left(x_{0}, y_{0} ; d y\right)$. For we have (omitting to write parentheses)

$K_{5} L_{0} K_{3} d y=K_{5} L_{0} K_{1} d y+K_{5} L_{0} K_{2} d y=K_{5} d y-K_{5} K_{4} d y=d y$, $K_{3} K_{5} L_{0} d y=K_{1} K_{5} L_{0} d y+K_{2} K_{5} L_{0} d y=K_{1}\left(K_{5}-K_{4} K_{5}\right) L_{0} d y=K_{1} L_{0} d y=d y$.

If now the constants $a$ and $b$ are sufficiently restricted, the conclusions $\left(C_{2}\right)$ and $\left(C_{3}\right)$ follow at once from Theorem IVI.

In case the hypothesis $\left(H_{5}\right)$ of the last theorem holds for every $(x, y, z)$ for which $d_{y} F$ has a reciprocal, we can derive from Theorem $\mathrm{V}^{\mathrm{I}}$ a theorem on the unique maximal sheet of solutions through a given solution. The statement and proof of this are fairly obvious. Here the sheet need not be singlevalued as it is in Theorem XI.

19. A special imbedding theorem. In this subsection we shall consider a case in which the spaces $\mathfrak{U}$ and $\mathfrak{B}$ are restricted to be euclidean spaces of $m$ and $k$ dimensions, respectively. The space $\mathfrak{X}$ need not be restricted. For the space $\mathfrak{Z}$ (which is to be of type $A_{0}$, as before, with $\sigma(p) \equiv 1$ ) we take the class of all functions $y$ on $\Re^{(0)}$ to $\mathfrak{B}$ which are bounded and measurable on $\Re^{(0)}$, i.e., all those functions $y$ whose components are bounded and measurable on $\Re^{(0)}$. The space $\mathbb{Z}$ corresponds to the space $\mathfrak{u}$ in exactly the same way. 
If distance is properly defined in the spaces $\mathfrak{U}$ and $\mathfrak{B}$, then $\mathfrak{V}$ and $\mathbb{Z}$ may be regarded as spaces of type $D$. For these spaces the integrals

$$
\int^{r} y(r) d r, \quad \int^{r} z(r) d r, \quad \int^{r}\|y(r)\| d r, \text { etc. }
$$

all exist in the Lebesgue sense, each integral being understood to be taken over the set of points in $\Re^{(0)}$ contained between the points $r_{0}$ and $r$ of $\Re^{(0)}$. In case the space $\Re$ enters twice as a component in the range of definition of a function, we shall use the notations $\Re^{\prime}$ and $r^{\prime}$ to denote the second component and second argument, respectively.

The form of the equations considered below was suggested by the paper of Hahn already mentioned.* However, our theorem is much more general than Hahn's.

TheOREM XIII. Consider a point $\left(x_{0}, y_{0}, z_{0}\right)$, a function $F$ on $\Re^{(0)}\left(x_{0}, y_{0}(r)\right.$, $\left.z_{0}(r)\right)_{c}$ to $\mathfrak{B}$, and a function $H$ on $\Re^{(0)} \Re^{\prime(0)}\left(x_{0}, y_{0}\left(r^{\prime}\right)\right)_{c}$ to $\mathfrak{U}$, with the following properties:

$\left(H_{1}\right) F$ and $H$ are bounded on their respective domains of definition;

$\left(H_{2}\right)$ for every point $(x, v, u)$ the function $F(r, x, v, u)$ is measurable in $r$ on every measurable set on which it is defined, and for every point $(x, v)$ the function $H\left(r, r^{\prime}, x, v\right)$ is measurable in $r$ and $r^{\prime}$ together and in $r^{\prime}$ alone, on every measurable set on which it is defined;

$\left(H_{8}\right) F$ is of class $\mathfrak{S}^{(n)}$ on $\left(x_{0}, y_{0}(r), z_{0}(r)\right)_{c}$ uniformly on $\Re^{(0)}\left(x_{0}, y_{0}(r)\right.$, $\left.z_{0}(r)\right)_{c}$, and $B$ is of class $\mathfrak{C}^{(n)}$ on $\left(x_{0}, y_{0}\left(r^{\prime}\right)\right)_{c}$ uniformly on $\Re^{(0)} \Re^{\prime(0)}\left(x_{0}, y_{0}\left(r^{\prime}\right)\right)_{c}$;

$\left(H_{4}\right)$ for every $r$ in $\Re^{(0)}$ we have

$$
\begin{aligned}
\int^{r} H\left(r, r^{\prime}, x_{0}, y_{0}\left(r^{\prime}\right)\right) d r^{\prime} & =z_{0}(r), \\
F\left(r, x_{0}, y_{0}(r), z_{0}(r)\right) & =v_{*} ;
\end{aligned}
$$

$\left(H_{5}\right)$ the functional determinant

$$
\left|F_{v}\left(r, x_{0}, y_{0}(r), z_{0}(r)\right)\right|
$$

(whose elements are the partial derivatives of the components of $F$ with respect to the components of $v$ ) is bounded away from zero on the set $\Re^{(0)}$.

Then there exist positive constants $a$ and $b$ and $a$ unique function $Y$ on $\left(x_{0}\right)_{b}$ to $\left(y_{0}\right)_{a}$ such that

$\left(C_{1}\right)$ for every $x$ in $\left(x_{0}\right)_{b}$ and $r$ in $\Re^{(0)}$ we have

* Monatshefte für Mathematik und Physik, vol. 14 (1903), p. 326. 


$$
F\left(r, x, Y(x \mid r), \int^{r} H\left(r, r^{\prime}, x, Y\left(x \mid r^{\prime}\right)\right) d r^{\prime}\right)=v_{*}
$$

$\left(C_{2}\right)$ the function $Y$ is of class $\mathfrak{C}^{(n)}$ on $\left(x_{0}\right)_{b}$ uniformly on $\left(x_{0}\right)_{b}$.

We wish to show first of all that for every point $(x, y, z)$ in $\left(x_{0}, y_{0}, z_{0}\right)_{c}$, the function $F(r, x, y(r), z(r))$ is bounded and measurable on $\Re^{(0)}$. This follows from $\left(H_{1}\right),\left(H_{2}\right)$ and $\left(H_{3}\right)$, and a theorem on the measurability of functions of measurable functions. ${ }^{*}$ Hence the function $F_{1}$ defined by

$$
F_{1}(x, y, z \mid r)=F(r, x, y(r), z(r))
$$

is on $\left(x_{0}, y_{0}, z_{0}\right)_{c}$ to $\mathfrak{Y}$. Similarly $H\left(r, r^{\prime}, x, y\left(r^{\prime}\right)\right)$ is bounded and measurable on $\Re^{(0)} \Re^{\prime(0)}$ and also is measurable on $\Re^{\prime(0)}$ for every $r$ in $\Re^{(0)}$. Then by Theorem III of Paper III the function $Z$ defined by

$$
Z(x, y \mid r)=\int^{r} H\left(r, r^{\prime}, x, y\left(r^{\prime}\right)\right) d r^{\prime}
$$

is on $\left(x_{0}, y_{0}\right)_{c}$ to 3 .

To show that the function $F_{1}$ is of class $\mathcal{C}^{(n)}$ on $\left(x_{0}, y_{0}, z_{0}\right)_{c}$ uniformly on $\left(x_{0}, y_{0}, z_{0}\right)_{c}$, we temporarily replace the spaces $\mathfrak{X}, \mathfrak{V}$, and $\mathbb{Z}$ by the more inclusive spaces of type $B$, consisting of all functions on $\Re^{(0)}$ to $\mathfrak{X}, \mathfrak{B}$, and $\mathfrak{u}$, respectively, which are bounded on $\Re^{(0)}$. Then we can apply Lemma 13.2 and Lemma 9.3.

To show that the function $Z$ is of class $\mathbb{E}^{(n)}$, we consider first the function $Z_{1}$ on $\left(x_{0}, y_{0}\right)_{c} \Re^{(0)}$ to $\mathfrak{u}$ defined by

$$
Z_{1}(x, y, r)=\int^{r} H\left(r, r^{\prime}, x, y\left(r^{\prime}\right)\right) d r^{\prime}=Z(x, y \mid r) .
$$

If we set $\mathfrak{I}=(\mathfrak{X}, \mathfrak{Z}), \mathfrak{B}=(\mathfrak{X}, \mathfrak{B})$, then it is readily verified that the function $d_{t} Z_{1}$ defined by

$$
d_{t} Z_{1}(x, y, r ; d x, d y)=\int^{r} d_{w} H\left(r, r^{\prime}, x, y\left(r^{\prime}\right) ; d x, d y\left(r^{\prime}\right)\right) d r^{\prime}
$$

satisfies the three conditions for the function $Z_{1}$ to be of class $\mathfrak{C}^{\prime}$ on $\left(x_{0}, y_{0}\right)_{c}$ uniformly on $\left(x_{0}, y_{0}\right)_{c} \Re^{(0)}$, with $d_{t} Z_{1}$ for its differential. The integrand on the right is measurable, since by Lemma 11.1 of Paper I each component is the limit of a convergent sequence of measurable functions.

The proof is completed by induction. Suppose that when $H$ is of class $\mathcal{E}^{(k)}, Z_{1}$ is of class $\mathbb{S}^{(k)}$, and suppose that $H$ is of class $\mathcal{S}^{(k+1)}$. Then $d_{w} H$ is

* See Paper III, Theorem II. The theorems of this note are simple extensions and consequences of a theorem in Carathéodory's Vorlesungen über reelle Funktionen, pp. 665-66. 
of class $\mathfrak{\complement}^{(k)}$ on $\left(x_{0}, y_{0}\left(r^{\prime}\right)\right)_{c}$ uniformly $\left(\Re^{(0)} \Re^{\prime(0)}\left(x_{0}, y_{0}\left(r^{\prime}\right)\right)_{c} \mathfrak{B} ;\|d w\|\right)$, and linear on $\mathfrak{B}$ uniformly on $\Re^{(0)} \Re^{\prime(0)}\left(x_{0}, y_{0}\left(r^{\prime}\right)\right)_{c}$. Hence by Lemma 7.2, $d_{w} H$ is of class $\mathfrak{E}^{(k)}$ on $\left(x_{0}, y_{0}\left(r^{\prime}\right)\right)_{c}\left(x_{*}, y_{*}\left(r^{\prime}\right)\right)_{c}$ uniformly on $\Re^{(0)} \Re^{\prime(0)}$ $\left(x_{0}, y_{0}\left(r^{\prime}\right)\right)_{c}\left(x_{*}, y_{*}\left(r^{\prime}\right)\right)_{c}$. This is a case exactly like the original, so that $d_{t} Z_{1}$ is of class $\mathbb{E}^{(k)}$ on $\left(x_{0}, y_{0}, x_{*}, y_{*}\right)_{c}$ uniformly on $\left(x_{0}, y_{0}, x_{*}, y_{*}\right)_{c} \Re^{(0)}$. Then by another application of Lemma 7.2 we find that $d_{t} Z_{1}$ is of class $\mathfrak{C S}^{(k)}$ on $\left(x_{0}, y_{0}\right)_{c}$ uniformly $\left(\left(x_{0}, y_{0}\right)_{c} \Re^{(0)} \mathfrak{T} ;\|d t\|\right)$, from which, by definition, $Z_{1}$ is of class ${ }^{\left({ }^{(k+1)}\right.}$. This completes the induction.

Finally, by application of Theorem I of $\S 4$, we have the result that the function $Z$ on $\left(x_{0}, y_{0}\right)_{c}$ to $Z$ is of class $\mathbb{C}^{(n)}$ on $\left(x_{0}, y_{0}\right)_{c}$ uniformly on $\left(x_{0}, y_{0}\right)_{c}$.

We have next to show that the differential $d_{y} F_{1}\left(x_{0}, y_{0}, z_{0} ; d y\right)$ has a reciprocal. From the hypothesis $\left(H_{3}\right)$, we can show that the elements of the matrix

$$
K_{1}(r) \equiv F_{v}\left(r, x_{0}, y_{0}(r), z_{0}(r)\right)
$$

are bounded on $\Re^{(0)}$, and from $\left(H_{2}\right)$ that they are measurable on $\Re^{(0)}$. Then the hypothesis $\left(H_{5}\right)$ shows that the matrix $K_{1}(r)$ has a reciprocal matrix $L_{1}(r)$, each of whose elements is bounded and measurable on $\Re^{(0)}$. Then the reciprocal $L_{0}$ of $d_{y} F_{1}\left(x_{0}, y_{0}, z_{0} ; d y\right)$ is obviously given by

$$
L_{0}(d y \mid r)=L_{1}(r) d y(r),
$$

where the multiplication on the right hand side is matrix multiplication.

Finally, it is readily verified that there exists a constant $k$ such that, for every $r$ in $\Re^{(0)}, d y$ in $\mathfrak{Y}$, and $d z$ in $\not$, we have

$$
\begin{aligned}
\left\|L_{0}(d y \mid r)\right\| & \leqq k\|d y(r)\|, \\
\left\|d_{y} Z\left(x_{0}, y_{0} ; d y \mid r\right)\right\| & =\left\|\int^{r} d_{v} H\left(r, r^{\prime}, x_{0}, y_{0}\left(r^{\prime}\right) ; d y\left(r^{\prime}\right)\right) d r^{\prime}\right\| \\
& \leqq k \int^{r}\left\|d y\left(r^{\prime}\right)\right\| d r^{\prime}, \\
\left\|d_{2} F_{1}\left(x_{0}, y_{0}, z_{0} ; d z \mid r\right)\right\| & =\left\|d_{u} F\left(r, x_{0}, y_{0}(r), z_{0}(r) ; d z(r)\right)\right\| \leqq k\|d z(r)\| .
\end{aligned}
$$

Thus all the hypotheses of Theorem XII are fulfilled, with $F$ replaced by $F_{1}$, and $H$ by $Z$, so that the theorem follows from Theorem XII.

In cases where the domains of definition of the functions $F$ and $H$ are sufficiently regular, and $F_{v}$ is always continuous on $\Re^{(0)}=$ a closed interval, it is easy to obtain from Theorem V of Paper I a theorem on the maximal sheet of solutions. We can then amplify the following Theorem XIV, by making use of Theorem $\mathrm{V}$ of $\S 10$. 
We next consider the case where the solution $Y(x)$ is continuous as a function of $r$, at the pcints of a set $\Re^{(1)}$ included in $\Re^{(0)}$. We have the following theorem:

THEOREM XIV. Suppose that the initial solution $y_{0}$, the functions $F$ and $H$, and the set $\Re^{(1)}$ contained in $\Re^{(0)}$ are such that

$\left(H_{1}\right) y_{0}$ is continuous in $r$ at the points of $\mathfrak{S R}^{(1)}$;

$\left(H_{2}\right)$ for every $(x, v, u), F$ is continuous in $r$ at the points of $\Re^{(1)}$ for which it is defined;

$\left(H_{3}\right) H$ is continuous in $r$ at the points of $\Re^{(1)}$ uniformly on $\Re^{\prime(0)}\left(x_{0}\right.$, $\left.y_{0}\left(r^{\prime}\right)\right)_{c}$.

Then the function $V$ on $\left(x_{0}\right)_{b} \Re^{(0)}$ to $\mathfrak{B}$ defined by

$$
V(x, r)=Y(x \mid r)
$$

is measurable on $\Re^{(0)}$ and continuous in $r$ at the points of $\Re^{(1)}$ for every $x$ in $\left(x_{0}\right)_{b}$, and is of class $\mathbb{E}^{(n)}$ on $\left(x_{0}\right)_{b}$ uniformly on $\left(x_{0}\right)_{b} \Re^{(0)}$.

To prove this theorem we apply Theorem IV of $\S 10$, with $\mathfrak{X}^{\prime}=\mathfrak{X}$, and $\mathfrak{y}^{\prime}=$ the subspace of $\mathfrak{Y}$ composed of all those functions $y$ which are continuous at the points of $\Re^{(1)}$. The subspace $\mathfrak{Y}^{\prime}$ is therefore a space of type $D_{1}$.

We have first to show that if the function $Z$ is defined by equation (19.1), and if the function $G$ on $\left(x_{0}, y_{0}\right)_{c}$ to $\mathfrak{V}$ is defined by

$$
G(x, y \mid r)=F(r, x, y(r), Z(x, y \mid r)),
$$

then $G$ as on $\left(x_{0}\right)_{b}\left(y_{0}\right)_{a}^{\prime}$ is to $\mathfrak{Y}^{\prime}$. Under the hypotheses of the present theorem, the function $Z$ as on $\left(x_{0}, y_{0}\right)_{c}$ is to $\eta^{\prime}$. From $\left(H_{3}\right)$ of Theorem XIII, and Lemma 12.2 of Paper I, we obtain the fact that $F$ is continuous on $\left(y_{0}(r), z_{0}(r)\right)_{c}$ uniformly on $\Re^{(0)}\left(x_{0}, y_{0}(r), z_{0}(r)\right)_{c}$. From this we readily obtain the desired property of the function $G$.

Secondly, we have to show that the reciprocal $L$ of $d_{y} G\left(x_{0}, y_{0} ; d y\right)$ as on $\mathfrak{Y}^{\prime}$ is to $\mathfrak{Y}^{\prime}$. We follow the steps in the proof of the existence of this reciprocal, as given in the proof of Theorem XII. The linear function $K_{2}$ on $\mathfrak{V}$ to $\mathfrak{Y}$ defined by

$$
K_{2}(d y \mid r)=d_{u} F\left(r, x_{0}, y_{0}(r), z_{0}(r) ; d_{y} Z\left(x_{0}, y_{0} ; d y \mid r\right)\right)
$$

is to $\mathfrak{Y}^{\prime}$, by Lemma 9.3. For the same reason the elements of the matrix $F_{v}\left(r, x_{0}, y_{0}(r), z_{0}(r)\right)$ are continuous in $r$ at the points of $\Re^{(1)}$. The same will consequently be true of the reciprocal matrix $L_{1}(r)$. Hence if the linear functions $L_{0}$ and $K_{4}$ are defined by

$$
L_{0}(d y \mid r)=L_{1}(r) d y(r), \quad K_{4}(d y)=-L_{0}\left(K_{2}(d y)\right),
$$


then $L_{0}$ and $K_{4}$ as on $\mathfrak{Y}^{\prime}$ are to $\mathfrak{Y}^{\prime}$. The reciprocal $K_{5}$ of $d y-K_{4}(d y)$ is given by the series

$$
K_{5}(d y)=\sum_{n=0}^{\infty} K_{4 n}(d y), \quad K_{40}(d y)=d y, \quad K_{4, n+1}(d y)=K_{4}\left(K_{4 n}(d y)\right) .
$$

Hence by Lemma $9.1, K_{5}$ as on $\mathfrak{Y}^{\prime}$ is to $\mathfrak{Y}^{\prime}$. Finally the function $L$, reciprocal to $d_{y} G\left(x_{0}, y_{0} ; d y\right)$, defined by

$$
L(d y)=K_{5}\left(L_{0}(d y)\right),
$$

evidently has the same property.

The last clause of the theorem follows immediately from Theorem I of $\$ 4$.

The imbedding theorem* for differential equations of the form

$$
F\left(r, z^{\prime}, z\right)=0
$$

becomes a special case of Theorems XIII and XIV if we set

$$
z^{\prime}=y, \quad z(r)=x+\int_{r_{0}}^{r} y\left(r^{\prime}\right) d r^{\prime}=x+\int_{r_{0}}^{r} H\left(y\left(r^{\prime}\right)\right) d r^{\prime} .
$$

\section{EXISTENCE THEOREMS FOR DIFFERENTIAL EQUATIONS}

Existence theorems for differential equations in general analysis have been given by $\mathrm{E}$. H. Moore and by T. H. Hildebrandt. $\dagger$ The theorems given here are somewhat different in form. One object of this section is to show how these existence theorems may be derived from the preceding imbedding and implicit function theorems. $f$

20. Notations. Instead of considering an arbitrary measurable set $\Re^{(0)}$, we now restrict attention to an open interval $\Re_{0}$ of the real axis. For the space $\mathfrak{V}$ we take the space (of type $C_{0}$ ) consisting of all functions $y$ on $\Re_{0}$ to $\mathfrak{B}$ which are continuous on $\Re_{0}$. We assume that the space $\mathfrak{B}$ is complete, so that the space $\mathfrak{Z}$ is also complete.

If we restrict attention to the points of a sub-interval $\Re_{0}^{1}$ of $\Re_{0}$, we obtain from the space $\mathfrak{Z}$ a space $\mathfrak{Y}^{1}$ consisting of functions $y^{1}$ on $\mathfrak{R}_{0}^{1}$ to $\mathfrak{B}$. The space $\mathfrak{V}^{1}$ is also a complete linear metric space of type $C_{0}$. Corresponding to $a_{\llcorner}^{7}$ region $\mathfrak{Z}_{0}$ of $\mathfrak{Z}$ there is a definitely determined region $\mathfrak{Z}_{0}^{1}$ of $\mathfrak{Z}^{1}$.

* Cf. Bolza, Vorlesungen über Variationsrechnung, pp. 179, 185.

Bliss, Bulletin of the American Mathematical Society, vol. 25 (1918), p. 15.

$\dagger$ Moore, Atti di IV Congresso (Rome, 1908), vol. II, p. 98.

Hildebrandt, these Transactions, vol. 18 (1917), p. 73.

$\ddagger$ Acknowledgements are due to Mr. H. B. Curry of Harvard University for suggestions on this subject. 
We let $r_{0}$ denote a point of the interval $\Re_{0}$, and we retain also the other notations of Part VI.

21. An existence theorem "im kleinen." We prove the following theorem:

THEOREM XV. Suppose the region $\mathfrak{Y}_{0}$ of $\mathfrak{Y}$, the point $y_{0}$ of $\mathfrak{Y}_{0}$, and the function $F$ on $\mathfrak{V}_{0}$ to $\mathfrak{Z}$ are such that

$\left(H_{1}\right)$ there is a positive constant $k$ such that, for every pair $y_{1}, y_{2}$ of points of $\mathfrak{Y}_{0}$ and every point $r$ of $\Re_{0}$ we have

$$
\left\|F\left(y_{1} \mid r\right)-F\left(y_{2} \mid r\right)\right\| \leqq k \int^{r}\left\|y_{1}(r)-y_{2}(r)\right\| ;
$$

$\left(H_{2}\right) \quad y_{0}\left(r_{0}\right)=F\left(y_{0} \mid r_{0}\right)$.

Then there exist an interval $\Re_{0}^{1}$ containing the point $r_{0}$, and a unique point $y^{1}$ in the corresponding region $\bigvee_{0}^{1}$, such that, for every $r$ of $\Re_{0}^{1}$ we have

$$
y^{1}(r)=F\left(y^{1} \mid r\right) .
$$

Moreover, $y^{1}\left(r_{0}\right)=y_{0}\left(r_{0}\right)$.

In order that the conclusion may have a meaning, the function $F(y \mid r)$, when $r$ is in $\Re_{0}^{1}$, must depend only on the part $y^{1}$ of $y$. That this is so follows from $\left(H_{1}\right)$, which in fact allows us to draw the conclusion that $F(y \mid r)$ depends only on the values of the function $y$ on the interval $\left(r_{0} r\right)$.

From the definition of a region, there is a neighborhood $\left(y_{0}\right)_{a}$ contained in $\mathfrak{D}_{0}$. Choose positive numbers $b<1$, and $c<(1-b) a$. Then the interval $\Re_{0}{ }^{1}$ is chosen so that, for every $r$ in $\Re_{0}{ }^{1}$, we have

$$
\begin{gathered}
k\left|r-r_{0}\right| \leqq b, \\
\left\|F\left(y_{0} \mid r\right)-F\left(y_{0} \mid r_{0}\right)\right\| \leqq \frac{c}{2}, \quad\left\|y_{0}\left(r_{0}\right)-y_{0}(r)\right\| \leqq \frac{c}{2} .
\end{gathered}
$$

Then the hypotheses of Theorem I of Paper I are fulfilled for the function $F$ on the neighborhood $\left(\left(y_{0}\right)_{a}\right)^{1}$.

To deduce the theorem from Theorem IX, we may omit the condition (21.1) on the interval $\Re_{0}^{1}$. The conditions (21.2) are retained, with the constant $c$ restricted only the inequality $0<c<a e^{-k d}$, where $d$ is the length of the interval $\Re_{0}$.

22. An existence theorem "im grossen." Consider a point $y_{0}$ of the space $\mathfrak{V}$, and a function $F$ on $\Re_{0} \mathfrak{B}_{r}$ to $\mathfrak{B}$, where $\mathfrak{B}_{r}=\left(y_{0}(r)\right)_{a}$. Suppose that for every 
$v, F$ is continuous on its domain of definition, and that for every $r$ and every $v_{1}$ and $v_{2}$ in $\mathfrak{B}_{r}$ we have

$$
\left\|F\left(r, v_{1}\right)-F\left(r, v_{2}\right)\right\| \leqq k\left\|v_{1}-v_{2}\right\| .
$$

Then we wish to show the existence of a continuous function $y$ on $\Re_{0}$ (or a part of $\Re_{0}$ ) to $\mathfrak{B}$, satisfying the equation

$$
y(r)=\int_{r_{0}}^{r} F(r, y(r)) d r+v,
$$

where $v$ is a point in $\left(y_{0}\left(r_{0}\right)\right)_{a}$. The integral here is a "Riemann" integral, and certainly exists if the integrand is continuous.* More particularly, we wish to show that a continuous solution $y$ exists on an interval $\Re_{0}^{1}$ included in $\Re_{0}$, such that, if $r_{1}$ is an end point of $\Re_{0}^{1}$ but not an end point of $\Re_{0}$, we have

$$
\lim _{r=r_{1}}\left\|y(r)-y_{0}(r)\right\|=a .
$$

To do this we can apply Theorem VIII of $\S 16$, i.e., define a function $G$ on $\Re_{0} \mathfrak{B}$ to $\mathfrak{B}$, equal to $F$ on $\Re_{0} \mathfrak{B}_{r}$, continuous on $\Re_{0}$ for every $v$, and satisfying the condition

$$
\left\|G\left(r, v_{1}\right)-G\left(r, v_{2}\right)\right\| \leqq 2 k\left\|v_{1}-v_{2}\right\|,
$$

for every $r, v_{1}, v_{2}$. Theorem VIII does not yield the continuity of the function $G$ on $\Re_{0}$. That $G$ is continuous on $\Re_{0}$ can be shown as follows. Let $\left\{b_{n}\right\}$ be a properly monotone increasing sequence of positive numbers approaching the number $a$. Let $G_{n}$ be the function formed (in the same way as $G$ ) from the function $F$ regarded as defined on $\Re_{0}\left(y_{0}(r)\right)_{b_{n}}$. Then each $G_{n}$ is continuous on $\Re_{0}$ for every $v$. Furthermore, we have $\left\|G(r, v)-G_{n}(r, v)\right\| \leqq k\left(a-b_{n}\right)$, so that $G_{n}$ approaches $G$ uniformly on $\Re_{0} \mathfrak{B}$.

If now we can show that the equation

$$
y(r)=\int_{r_{0}}^{r} G(r, y(r)) d r+v
$$

has a unique continuous solution, then the part of it falling in the domain of definition of the function $F$ is the solution of equation (22.1) which we desire. The integrand $G(r, y(r))$ above is evidently continuous on $\Re_{0}$ whenever $y$ is continuous on $\Re_{0}$, so that the integral surely exists for every $y$ in the space $\mathfrak{Y}$. The existence of a solution of (22.2) is shown in the following theorem.

* See Paper II, Theorem I. 
Theorem XVI. Suppose the function $F$ on $\mathfrak{Y}$ to $\mathfrak{Y}$ has the property that there exists a positive constant $k$ such that, for every $y_{1}$ and $y_{2}$ in $\mathfrak{Y}$ and every $r$ in $\Re_{0}$ we have

$$
\left\|F\left(y_{1} \mid r\right)-F\left(y_{2} \mid r\right)\right\| \leqq k \int^{r}\left\|y_{1}(r)-y_{2}(r)\right\| .
$$

Then there is a unique function $y$ in $\mathfrak{Y}$ such that

$$
y=F(y) .
$$

This theorem follows immediately from Theorem IX. The initial function $y_{0}$ may be chosen arbitrarily. Then take $a>e^{k d}\left\|F\left(y_{0}\right)-y_{0}\right\|$, and the solution lies in $\left(y_{0}\right)_{a}$.

23. A special case.* We wish now to derive an existence theorem (im kleinen) for solutions of equations of the form discussed in $\S 19$. Let $\mathfrak{U}$ and $\mathfrak{B}$ be ordinary spaces of $m$ and $k$ dimensions, respectively. As before $\Re_{0}$ and $\Re_{0}^{\prime}$ denote the same linear interval, and $r_{0}$ denotes a point in it.

Theorem XVII. Consider a function $F$ on $\Re_{0} \mathfrak{B}_{0} \mathfrak{U}_{0}$ to $\mathfrak{B}$, a function $B$ on $\mathfrak{R}_{0} \Re_{0}^{\prime} \mathfrak{B}_{0}$ to $\mathfrak{U}$, and a point $v_{0}$ of $\mathfrak{B}_{0}$, with the following properties:

$\left(H_{1}\right) \mathfrak{u}_{0}$ contains the origin $u_{*}$;

( $\left.H_{2}\right) F\left(r_{0}, v_{0}, u_{*}\right)=v_{*}$;

$\left(H_{3}\right) F$ is of class $\mathfrak{S}^{\prime}$ on $\mathfrak{B}_{0} \mathfrak{U}_{0}$ uniformly on $\Re_{0} \mathfrak{B}_{0} \mathfrak{U}_{0}$, and continuous on $\Re_{0}$;

$\left(H_{4}\right) H$ is bounded on $\Re_{0} \Re_{0}^{\prime} \mathfrak{B}_{0}$, of class $\mathbb{E}^{\prime}$ on $\mathfrak{B}_{0}$ uniformly on $\Re_{0} \Re_{0}^{\prime} \mathfrak{B}_{0}$, continuous on $\Re_{0}$ uniformly on $\Re_{0}^{\prime} \mathfrak{B}_{0}$, and measurable on $\Re_{0}^{\prime}$;

$\left(H_{5}\right)$ the functional determinant

$$
\left|F_{v}\left(r_{0}, v_{0}, u_{*}\right)\right|
$$

is different from zero.

Then there exist an interval $\Re_{0}^{1}$ containing $r_{0}$, a positive constant $a$, and a unique continuous function $y^{1}$ on $\Re_{0}^{1}$ to $\left(v_{0}\right)_{a}$ such that, for every $r$ in $\Re_{0}^{1}$,

$$
F\left(r, y^{1}(r), \int_{r_{0}}^{r} H\left(r, r^{\prime}, y^{1}\left(r^{\prime}\right)\right) d r^{\prime}\right)=v_{*} .
$$

Moreover, $y^{1}\left(r_{0}\right)=v_{0}$.

The first step in the proof is to apply the implicit function theorems to the equation $F(r, v, u)=v_{*}$ to show that there exist positive constants $a$ and $b$, and a unique solution $V(r, u)$ on $\left(r_{0}, u_{*}\right)_{b}$ to $\left(v_{0}\right)_{a}$, and that the function $V$ so obtained is continuous on $\left(r_{0}\right)_{b}$, and is of class $\mathbb{E}^{\prime}$ on $\left(u_{*}\right)_{b}$ uniformly

* Cf. Hahn, loc. cit. 
on $\left(r_{0}, u_{*}\right)_{b}$. The simplest procedure seems to be to apply Theorem III of Paper $I$ to show the existence and uniqueness of the solution $V$. The continuity of $V$ on $\left(r_{0}, u_{*}\right)_{b}$ is then obtained by Theorem II of Paper I. From $\left(H_{3}\right)$ and the statement just made we can show that the functional determinant

$$
\left|F_{v}(r, V(r, u), u)\right|
$$

is continuous on the neighborhood $\left(r_{0}, u_{*}\right)_{b}$. Hence if $b$ is sufficiently small, the functional determinant (23.1) is bounded away from zero on that neighborhood, by $\left(H_{5}\right)$. Therefore we can apply Theorem VI of $\S 14$ to show that (when $b$ is sufficiently small) $V$ is of class $\mathbb{E}^{\prime}$ on $\left(u_{*}\right)_{b}$ uniformly on $\left(r_{0}, u_{*}\right)_{b}$.

We next consider the function $H$. First, $H\left(r, r^{\prime}, y^{1}\left(r^{\prime}\right)\right)$ is measurable in $r^{\prime}$ for every continuous function $y^{1}$ on $\left(r_{0}\right)_{b}$ to $\mathfrak{B}$ which is interior to $\mathfrak{B}_{0}$. This follows from Theorem II of Paper III. Secondly, the function

$$
U\left(r, y^{1}\right)=\int_{r_{0}}^{r} H\left(r, r^{\prime}, y^{1}\left(r^{\prime}\right)\right) d r^{\prime}
$$

is continuous on $\left(r_{0}\right)_{b}$.

For the initial function $y_{0}^{1}$ on $\left(r_{0}\right)_{b}$ to $\mathfrak{B}$ we take $y_{0}^{1}(r)=v_{0}$ for every $r$. Let

$$
G\left(y^{1} \mid r\right)=V\left(r, U\left(r, y^{1}\right)\right) .
$$

Then $G$ is on $\left(y_{0}^{1}\right)_{a}$ to $\mathfrak{Y}^{1}$. By two applications of Taylor's theorem we find

$$
\left\|G\left(y_{1}{ }^{1} \mid r\right)-G\left(y_{2}{ }^{1} \mid r\right)\right\| \leqq M^{2} \int_{r_{0}}^{r}\left\|y_{1}{ }^{1}\left(r^{\prime}\right)-y_{2}{ }^{1}\left(r^{\prime}\right)\right\| d r^{\prime},
$$

where $M$ is greater than the moduli of $d_{u} V$ and $d_{v} H$. Hence we can apply Theorem XV to secure the desired result, with possibly a further restriction on the interval $\left(r_{0}\right)_{b}=\Re_{0}^{1}$, and consequent alteration of $Y^{1}$.

VIII. SPECIAL PROPERTIES OF LINEAR EQUATIONS

24. In this section we seek to generalize the fundamental property of solutions of systems of homogeneous linear differential equations of the first order which states that a set of solutions linearly independent at a given point are linearly independent at every point of their interval of existence.

As a basis for deriving this property, we state an elegant theorem relating to a certain class of linear transformations defined by a linear equation. This theorem is due to Professor T. H. Hildebrandt, although the proof for the more general case stated below is my own. 
Let $\Re^{(0)}$ be a bounded and measurable linear set of the real axis $\Re$. Let $\mathfrak{B}$ be the complete linear metric space of type $B$ consisting of all functions $v$ on a range $\mathfrak{B}$ to $\Re$ which are bounded on $\mathfrak{B}$. Let $\mathfrak{V}$ be the complete linear metric space of type $A_{0}$ consisting of all functions $y$ on $\Re^{(0)}$ to $\mathfrak{B}$ which are bounded on $\Re^{(0)}$, and are such that $y(r \mid p)$ is measurable on $\Re^{(0)}$ for every $p$.

THEOREM XVIII. Let $K$ be a function on $\mathfrak{B} \Re^{(0)}$ to $\mathfrak{B}$ which is linear on $\mathfrak{B}$ uniformly on $\Re^{(0)}$, and such that the function $K_{1}$ on $\mathfrak{V}$ defined by

$$
K_{1}(y \mid r)=K(y(r), r)
$$

is to $\mathfrak{Y}$. Then there exists a unique function $V$ on $\mathfrak{B} \Re^{(0)} \Re^{(0)}$ to $\mathfrak{B}$ such that

$\left(C_{1}\right)$ for each $v$ and $r_{1}, V\left(v, r_{1}, r\right)$, as a function of $r$ on $\Re^{(0)}$, belongs to the class $\mathfrak{Y}$;

$\left(C_{2}\right)$ for every $v$ in $\mathfrak{B}$, and every $r_{1}$ and $r$ in $\Re^{(0)}$, we have

$$
V\left(v, r_{1}, r\right)=\int_{r_{1}}^{r} K\left(V\left(v, r_{1}, r^{\prime}\right), r^{\prime}\right) d r^{\prime}+v,
$$

where the integral is taken over the points of $\Re^{(0)}$ contained in the interval $\left(r_{1} r\right)$.

This function $V$ has the further properties

$\left(C_{3}\right) \quad V$ is linear on $\mathfrak{B}$ uniformly on $\Re^{(0)} \Re^{(0)}$;

$\left(C_{4}\right)$ for every $v$ in $\mathfrak{B}$, and every $r_{1}, r_{2}, r_{3}$ in $\Re^{(0)}$ we have

$$
V\left(v, r_{1}, r_{3}\right)=V\left(V\left(v, r_{1}, r_{2}\right), r_{2}, r_{3}\right) ;
$$

$\left(C_{6}\right)$ for every $r_{1}, r_{2}$ in $\Re^{(0)}$ the linear function $V\left(v, r_{1}, r_{2}\right)$ has a reciprocal, viz., $V\left(v, r_{2}, r_{1}\right)$.

The conclusions $\left(C_{1}\right),\left(C_{2}\right)$, and $\left(C_{3}\right)$ follow at once from Theorem $\mathrm{X}$ of $\S 18$. To obtain $\left(C_{4}\right)$ we have

$$
\begin{aligned}
V\left(v, r_{1}, r\right)= & \int_{r_{2}}^{r} K\left(V\left(v, r_{1}, r^{\prime}\right), r^{\prime}\right) d r^{\prime} \\
& +\int_{r_{1}}^{r_{2}} K\left(V\left(v, r_{1}, r^{\prime}\right), r^{\prime}\right) d r^{\prime}+v \\
= & \int_{r_{2}}^{r} K\left(V\left(v, r_{1}, r^{\prime}\right), r^{\prime}\right) d r^{\prime}+V\left(v, r_{1}, r_{2}\right) .
\end{aligned}
$$

Then since the equation$$
y(r)=\int_{r_{2}}^{r} K\left(y\left(r^{\prime}\right), r^{\prime}\right) d r^{\prime}+V\left(v, r_{1}, r_{2}\right)
$$

has a unique solution, viz., $y(r)=V\left(V\left(v, r_{1}, r_{2}\right), r_{2}, r\right)$, we have the required result. $\left(C_{5}\right)$ is an obvious corollary of $\left(C_{4}\right)$. 
The proof just given for the property $\left(C_{4}\right)$ is valid on the interval of existence of the solution, whether the function $K$ is linear or not. The existence and uniqueness may be obtained from Theorem XVI of $\S 22$. When $K$ is linear, another proof of the property $\left(C_{4}\right)$ may be given by using formulas analogous to (24.1) for the terms of the infinite series obtained by the method of successive approximations, of which the function $V$ is the sum.

THEOREM XIX. Let $K_{1}, K_{2}$, and $L_{1}$ be linear functions on $\mathfrak{B} \Re^{(0)}$ to $\mathfrak{B}$ with the same properties as the function $K$ of Theorem XVIII, and let $L_{1}$ be reciprocal to $K_{1}$, for every $r$ of $\Re^{(0)}$. Then there exists a unique function $Y$ on $\mathfrak{B}^{(0)}$ to V) such that

$\left(C_{1}\right)$ for every $v, r_{1}$, and $r$, we have

$$
K_{1}\left(Y\left(v, r_{1} \mid r\right), r\right)=\int_{r_{1}}^{r} K_{2}\left(Y\left(v, r_{1} \mid r^{\prime}\right), r^{\prime}\right) d r^{\prime}+v .
$$

The function $Y$ has the further properties

$\left(C_{2}\right) Y$ is linear on $\mathfrak{B}$ uniformly on $\Re^{(0)}$;

$\left(C_{3}\right)$ if $Y\left(v, r_{1} \mid r_{2}\right)=v_{*}$ for a certain $r_{1}, r_{2}$, then $v=v_{*}$;

$\left(C_{4}\right)$ if the points $v_{1}, v_{2}, \cdots, v_{n}$ are linearly independent, so are the points

$$
Y\left(v_{1}, r_{1} \mid r_{2}\right), Y\left(v_{2}, r_{1} \mid r_{2}\right), \cdots, Y\left(v_{n}, r_{1} \mid r_{2}\right),
$$

for every $r_{1}$ and $r_{2} .{ }^{*}$

Under the transformation of $\mathfrak{Z}$ into $\mathfrak{Z}$ defined by

the equations

$$
y^{\prime}(r)=K_{1}(y(r), r), \quad y(r)=L_{1}\left(y^{\prime}(r), r\right),
$$

$$
\begin{aligned}
K_{1}(y(r), r) & =\int_{r_{1}}^{r} K_{2}\left(y\left(r^{\prime}\right), r^{\prime}\right) d r^{\prime}+v \\
y^{\prime}(r) & =\int_{r_{1}}^{r} K_{2}\left(L_{1}\left(y^{\prime}\left(r^{\prime}\right), r^{\prime}\right), r^{\prime}\right) d r^{\prime}+v
\end{aligned}
$$

are equivalent. Then if we set

$$
K(v, r)=K_{2}\left(L_{1}(v, r), r\right), \quad Y\left(v, r_{1} \mid r\right)=L_{1}\left(V\left(v, r_{1}, r\right), r\right),
$$

we have our theorem from Theorem XVIII. We obtain the property $\left(C_{3}\right)$ from the fact that both $L_{1}$ and $V$ have reciprocals, and $\left(C_{4}\right)$ follows from $\left(C_{3}\right)$ and the linearity of $Y$.

* Cf. Hahn, loc. cit., pp. 330-332.

University of Chicago, Chicago, ill. 